\title{
Contrasting gene expression patterns in grain of high and low asparagine wheat genotypes in response to sulphur supply
}

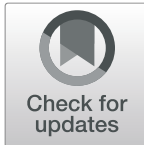

Tanya Y. Curtis ${ }^{1,3}$, Sarah Raffan ${ }^{1}$, Yongfang Wan ${ }^{1}$, Robert King ${ }^{2}$, Asier Gonzalez-Uriarte ${ }^{2,4}$ and Nigel G. Halford ${ }^{1 *}$ (D)

\begin{abstract}
Background: Free asparagine is the precursor for acrylamide formation during cooking and processing of grains, tubers, beans and other crop products. In wheat grain, free asparagine, free glutamine and total free amino acids accumulate to high levels in response to sulphur deficiency. In this study, RNA-seq data were acquired for the embryo and endosperm of two genotypes of bread wheat, Spark and SR3, growing under conditions of sulphur sufficiency and deficiency, and sampled at 14 and 21 days post anthesis (dpa). The aim was to provide new knowledge and understanding of the genetic control of asparagine accumulation and breakdown in wheat grain.

Results: There were clear differences in gene expression patterns between the genotypes. Sulphur responses were greater at $21 \mathrm{dpa}$ than $14 \mathrm{dpa}$, and more evident in SR3 than Spark. TaASN2 was the most highly expressed asparagine synthetase gene in the grain, with expression in the embryo much higher than in the endosperm, and higher in Spark than SR3 during early development. There was a trend for genes encoding enzymes of nitrogen assimilation to be more highly expressed in Spark than SR3 when sulphur was supplied. TaASN2 expression in the embryo of SR3 increased in response to sulphur deficiency at $21 \mathrm{dpa}$, although this was not observed in Spark. This increase in TaASN2 expression was accompanied by an increase in glutamine synthetase gene expression and a decrease in asparaginase gene expression. Asparagine synthetase and asparaginase gene expression in the endosperm responded in the opposite way. Genes encoding regulatory protein kinases, SnRK1 and GCN2, both implicated in regulating asparagine synthetase gene expression, also responded to sulphur deficiency. Genes encoding bZIP transcription factors, including Opaque2/bZIP9, SPA/bZIP25 and BLZ1/OHP1/bZIP63, all of which contain SnRK1 target sites, were also expressed. Homeologues of many genes showed differential expression patterns and responses, including TaASN2.
\end{abstract}

Conclusions: Data on the genetic control of free asparagine accumulation in wheat grain and its response to sulphur supply showed grain asparagine levels to be determined in the embryo, and identified genes encoding signalling and metabolic proteins involved in asparagine metabolism that respond to sulphur availability.

Keywords: Asparagine synthetase, Amino acid metabolism, Acrylamide, bZIP, Crop composition, Food safety, RNAseq, Sulphur, Triticum aestivum, Wheat

\footnotetext{
* Correspondence: nigel.halford@rothamsted.ac.uk

${ }^{1}$ Plant Sciences Department, Rothamsted Research, Harpenden, Hertfordshire

AL5 2JQ, UK

Full list of author information is available at the end of the article
}

(c) The Author(s). 2019 Open Access This article is distributed under the terms of the Creative Commons Attribution 4.0 International License (http://creativecommons.org/licenses/by/4.0/), which permits unrestricted use, distribution, and reproduction in any medium, provided you give appropriate credit to the original author(s) and the source, provide a link to the Creative Commons license, and indicate if changes were made. The Creative Commons Public Domain Dedication waiver (http://creativecommons.org/publicdomain/zero/1.0/) applies to the data made available in this article, unless otherwise stated. 


\section{Background}

Interest in the synthesis, accumulation and breakdown of asparagine in crop plants has been reinvigorated in recent years due to the discovery that free (soluble, non-protein) asparagine is the precursor for acrylamide formation during cooking and processing [1-3] and its concentration is the main determinant of acrylamide-forming potential in wheat and other cereals [4-8]. Acrylamide forms in the Maillard reaction, which also requires reducing sugars, such as glucose, fructose and maltose, but the carbon skeleton of the acrylamide that forms is derived from free asparagine.

Fried, baked, roasted and toasted cereal, coffee and potato products are the main sources of dietary acrylamide. Acrylamide is classed as a Group 2a human carcinogen [9] and in 2015 the European Food Safety Authority (EFSA) Expert Panel on Contaminants in the Food Chain (CONTAM) issued a report concluding that the margins of exposure to dietary acrylamide indicated 'a concern for neoplastic effects' [10]. Subsequently (April 2018), Commission Regulation (EU) 2017/2158 came into force across the European Union, introducing compulsory risk management measures that apply to all food businesses [11].

The development of crop varieties with reduced acrylamide-forming potential may enable the food industry to comply with regulations without costly changes to manufacturing lines or reduced product quality. In the case of cereals, this means varieties with reduced and more consistent free asparagine concentration in the grain. There is, therefore, a need for greater knowledge and understanding of the genetic control of asparagine synthesis, accumulation and breakdown.
Asparagine is synthesised through the ATPdependent transfer of the amino group of glutamine to a molecule of aspartate to generate glutamate and asparagine, a reaction catalysed by the enzyme asparagine synthetase. An extensive network comprising genes, enzymes, transcription factors and regulatory proteins has been constructed [12] and the components of the core of that network are shown in Fig. 1. Free asparagine accumulates in many plant tissues in response to a range of abiotic and biotic stresses, as well as during normal physiological processes such as seed germination [13]. In wheat grain, it accumulates to very high levels in response to sulphur deficiency [4-7] and poor disease control [14]. Sulphur deficiency also brings about large increases in free glutamine and total free amino acid concentrations $[6,15]$. There are also substantial differences in the free asparagine concentration of grain from different wheat varieties and genotypes [15].

In the present study, RNA-seq analysis was used to compare two wheat genotypes, variety Spark and a doubled haploid line, SR3, from a Spark $\times$ Rialto mapping population [16]. SR3 has previously been shown to have a lower concentration of free asparagine in the grain than Spark (1.68 versus $2.71 \mathrm{mmol}$ per $\mathrm{kg}$ when grown in compost, a difference of $61 \%$ with respect to the lower figure, and 2.05 versus $2.54 \mathrm{mmol}$ per $\mathrm{kg}$ when grown in vermiculite, a difference of $24 \%$ with respect to the lower figure [6]). It also has a lower concentration of total free amino acids, particularly under conditions of sulphur deficiency [6]. The two genotypes were grown under conditions of sulphur sufficiency and deficiency, and the

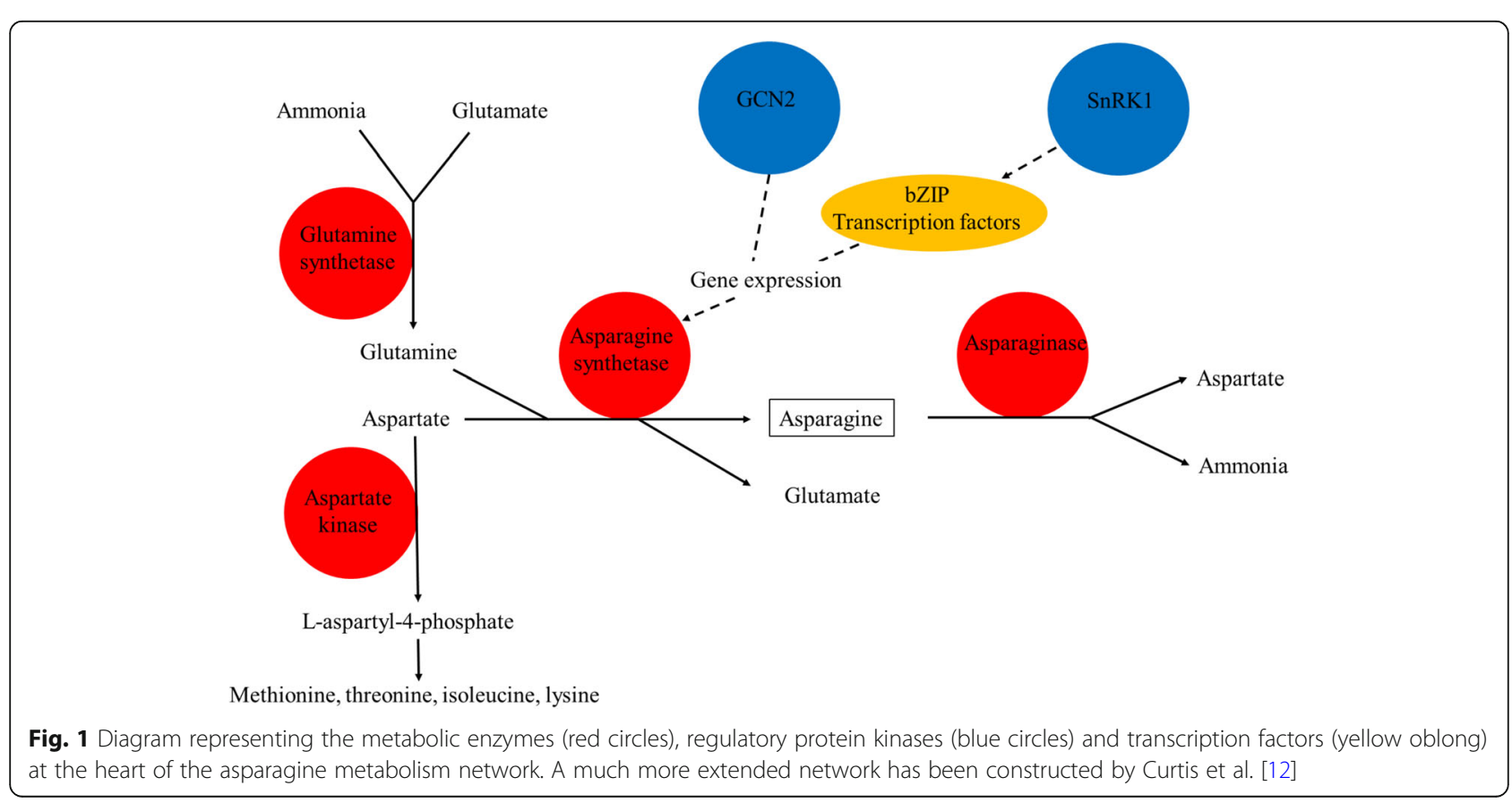


data analysed to identify the genes involved in the response to sulphur deficiency and how the sulphur response differed between the two genotypes, focussing on genes involved in asparagine metabolism and its regulation, and nitrogen assimilation.

\section{Results}

Winter wheat genotypes SR3 and Spark were grown in a glasshouse with and without sulphur supplied and grain samples taken at 14 and 21 days post-anthesis (dpa). The embryo and endosperm were separated, and four embryo and four endosperm samples (biological replicates) were analysed for each genotype, time-point and treatment, making a total of 64 samples from which RNA was prepared for RNA-seq analysis. One sample for each of the genotypes was discarded because the RNA was not of adequate quality.

\section{Exploratory analyses}

A principal component analysis (PCA) was performed and the resulting plots are shown in Fig. 2. The PCA showed the main source of variation to be tissue (Fig. 2a), as would be expected. This plot identified two samples that seemed to have been mislabelled, and these were excluded from further analysis. The secondary source of variation was the genotype (Fig. 2b), followed by treatment ( $\mathrm{S}+$ versus S-; Fig. 2c) and time (14 dpa versus $21 \mathrm{dpa}$ (Fig. 2d). In addition, the analysis showed one batch of data to be slightly different from a second (Fig. 2e). Three further samples were dropped due to poor clustering, reducing the number of samples to 57 . The raw data have been deposited in European Nucleotide Archive (ENA) and is publicly available (https://www.ebi.ac.uk/ena) using the study accession: PRJEB31122.

The data were analysed using the DESeq2 $\mathrm{r}$ package to make pairwise comparisons of high versus low sulphur for each of the eight genotype $\times$ timepoint $\times$ treatment combinations. Genes were classified as differentially expressed using an adjusted $p$-value of $<0.05$. Comparisons were made between high and low sulphur treatments in the two genotypes, at different stages and in the two tissue types (Table 1A). Overall, more genes showed differential expression in response to the treatment in SR3 than Spark, and there was a much greater response by $21 \mathrm{dpa}$ than $14 \mathrm{dpa}$. There were also more genes changing in the endosperm than the embryo, particularly in Spark (only two genes showed differential expression in Spark embryos, even at $21 \mathrm{dpa}$ ). Table $1 \mathrm{~B}$ contrasts the responses in the embryo and endosperm and shows that a very different set of genes changed in the two tissues, with only a relatively small number of genes responding in both. Overall, the numbers of genes responding to sulphur were much higher than those reported by Yu et al. [17].
Functional enrichment analysis was performed separately for the differentially expressed gene lists. Three of the pairwise comparisons (variety Spark, endosperm, 14 dpa; embryo, 14 and $21 \mathrm{dpa}$ ) did not show enrichment because of the small number of differentially expressed genes. The results of the enrichment of the rest of the comparisons are given in Additional file 1.

\section{Confirmatory analyses}

The validity of the data with respect to tissue type was confirmed by checking the expression of genes encoding prolamin storage proteins, which are known to be expressed endosperm-specifically [18]. These all showed clear, endosperm-specific expression, and this is shown graphically for a gene encoding a low molecular weight glutenin subunit in Fig. 3a. In contrast, the late embryogenesis abundant 12 (LEA12) gene was expressed at much higher levels in the embryo than the endosperm, as is typical of this class of genes (see [19] for review) (Fig. 3b). The relatively very high expression of the low molecular weight glutenin subunit gene is also in line with expectations [18].

Several genes are known to be responsive to sulphur feeding in wheat, but this has usually been demonstrated in seedlings rather than grain tissues [20]. Nevertheless, there were examples in the data of genes responding clearly to sulphur availability in the expected manner, and this is shown for an ethylene-insensitive 3-like 5 (EIL5) gene [21] in Fig. 3c. This gene was clearly induced by sulphur feeding in the endosperm at $21 \mathrm{dpa}$, although the response was not significant in the embryo at $21 \mathrm{dpa}$ and was not apparent in either tissue at $14 \mathrm{dpa}$, showing the sulphur response to be much more evident at $21 \mathrm{dpa}$ than $14 \mathrm{dpa}$. The reference numbers for these three genes are provided in Additional file 2.

\section{Genes of interest in asparagine synthesis, accumulation and breakdown}

The aim of the study was to gain an understanding of the genetic factors responsible for the difference in free asparagine and total free amino acid concentration in the grain of Spark and SR3, and the factors involved in changes in free asparagine and total free amino acid accumulation in response to sulphur availability. The data on genes included in the network identified by Curtis et al. [12] as being involved in asparagine synthesis, turnover and accumulation were therefore analysed in detail.

\section{Asparagine synthetases}

Wheat (Triticum aestivum) contains four asparagine synthetase genes, called TaASN1-TaASN4 [22]. The enzymes encoded by these genes are very similar to each other, with molecular masses between 65 and $67 \mathrm{kDa}$. Heterologous expression and biochemical characterisation of TaASN1 

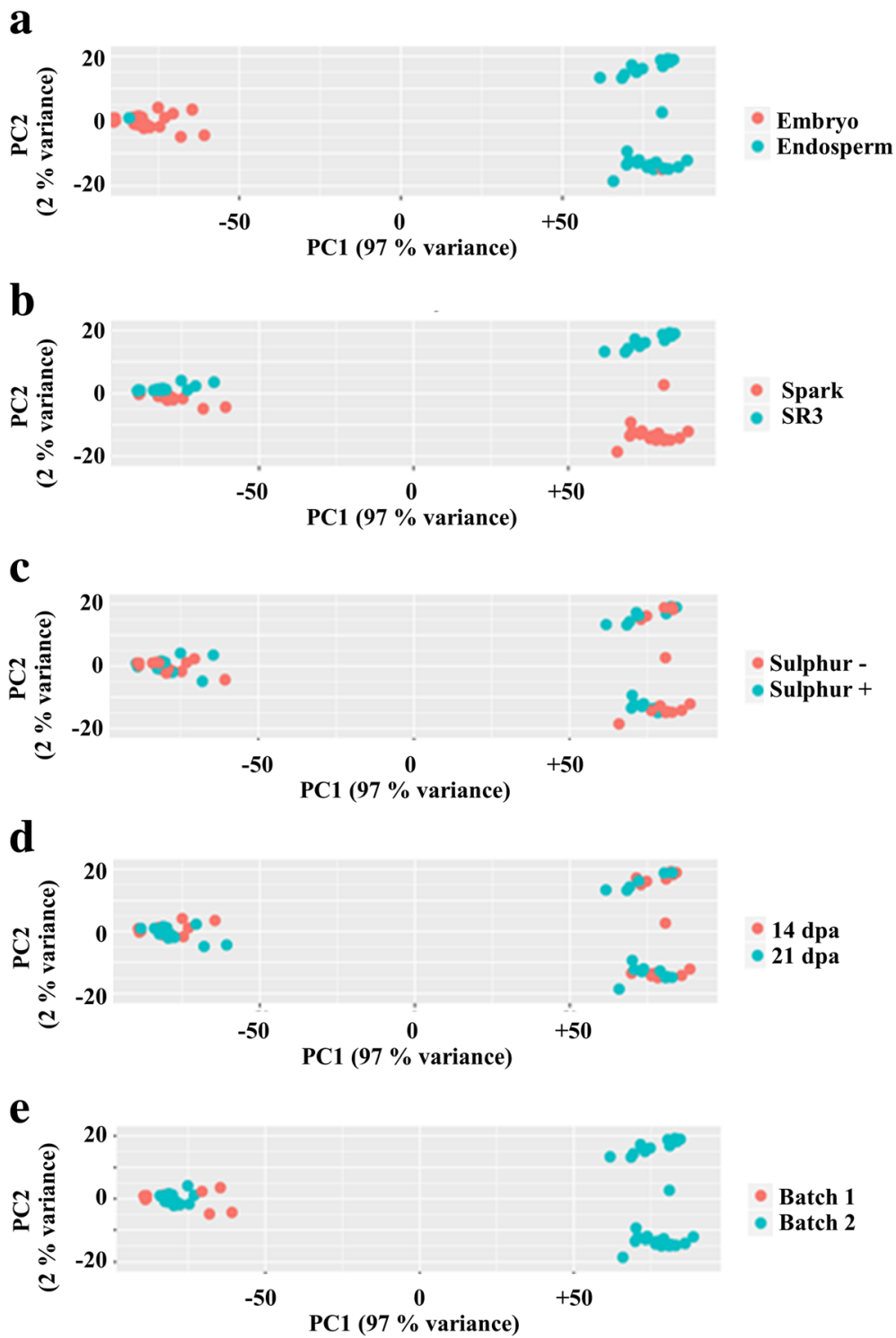

Fig. 2 Plots derived from Principal Component analysis (PCA) of the data showing the variance due to: a Tissue. b Genotype. c Sulphur treatment. d Timepoint. e Batch

and TaASN2 enzymes also showed them to have similar biochemical properties [23]. TaASN1, TaASN2 and TaASN4 are all single copy genes, located on chromosomes 5, 3 and 4, respectively, of each genome (A, B and D), although some varieties lack a TaASN2 gene in the B genome [23]. The ASN1 and ASN2 genes of durum pasta wheat (Triticum turgidum ssp. durum) are also located on chromosomes 5 and 3, respectively [24]. Two copies of TaASN3, TaASN3.1 and TaASN3.2, are present on chromosome 1 of each genome. TaASN3.2 is annotated as TaASN5 in some databases, but the similarities in gene structure and chromosomal location of TaASN3.1 and TaASN3.2 justify regarding them as two copies of the same gene. Gao et al. [22] examined the expression of
TaASN1, TaASN2 and TaASN3 and showed TaASN2 to be the most highly expressed in the grain. However, TaASN1 was the most responsive to nitrogen availability and sulphur deficiency and had previously been shown to respond to salt stress, osmotic stress and ABA [25]. The ASN1 gene of durum wheat has also been shown to respond to nitrogen availability [24].

Figure 4 shows the expression of all the TaASN genes in the present study, comparing the expression in Spark with SR3 in the embryo and endosperm for each homeologue separately, with and without sulphur supplied, at 14 dpa (Fig. 4a and b) and $21 \mathrm{dpa}$ (Fig. 4c and d). The reference numbers for the genes are provided in Additional file 2. The data revealed much higher total asparagine synthetase 
Table 1 Summary of the changes in gene expression in response to sulphur. A. The number of genes that showed significant $(p<$ 0.05) changes in expression in response to sulphur deficiency, in total and split into upregulated and downregulated. B. As for A, but contrasting the gene expression changes between tissue types

\begin{tabular}{|c|c|c|c|c|c|c|c|c|c|c|c|c|}
\hline \multicolumn{13}{|l|}{ A } \\
\hline & \multicolumn{6}{|c|}{21 days } & \multicolumn{6}{|c|}{14 days } \\
\hline & \multicolumn{3}{|c|}{ Endosperm } & \multicolumn{3}{|c|}{ Embryo } & \multicolumn{3}{|c|}{ Endosperm } & \multicolumn{3}{|c|}{ Embryo } \\
\hline & Total & Up & Down & Total & Up & Down & Total & Up & Down & Total & Up & Down \\
\hline Spark Total & 5151 & 2422 & 2729 & 2 & 2 & 0 & 1 & 0 & 1 & 0 & 0 & 0 \\
\hline SR3 Total & 8905 & 3179 & 5726 & 5223 & 1901 & 3322 & 869 & 344 & 525 & 646 & 633 & 13 \\
\hline In both (Spark) & 1707 & 712 & 995 & 0 & 0 & 0 & 0 & 0 & 0 & 0 & 0 & 0 \\
\hline In both (SR3) & 1707 & 659 & 1048 & 0 & 0 & 0 & 0 & 0 & 0 & 0 & 0 & 0 \\
\hline Spark only & 3444 & 1710 & 1734 & 2 & 0 & 2 & 1 & 0 & 1 & 0 & 0 & 0 \\
\hline SR3 only & 7198 & 2520 & 4678 & 5223 & 1901 & 3322 & 869 & 344 & 525 & 646 & 633 & 13 \\
\hline \multicolumn{13}{|l|}{ B } \\
\hline & \multicolumn{6}{|c|}{21 days } & \multicolumn{6}{|c|}{14 days } \\
\hline & \multicolumn{3}{|l|}{ Spark } & \multicolumn{3}{|l|}{ SR3 } & \multicolumn{3}{|l|}{ Spark } & \multicolumn{3}{|l|}{ SR3 } \\
\hline & Total & Up & Down & Total & Up & Down & Total & Up & Down & Total & Up & Down \\
\hline Endosperm & 5151 & 2422 & 2729 & 8905 & 3179 & 5726 & 1 & 0 & 1 & 869 & 344 & 525 \\
\hline Embryo & 2 & 2 & 0 & 5223 & 1901 & 3322 & 0 & 0 & 0 & 646 & 330 & 316 \\
\hline In both (end) & 1 & 1 & 0 & 951 & 322 & 629 & 0 & 0 & 0 & 27 & 27 & 0 \\
\hline In both (emb) & 1 & 1 & 0 & 951 & 421 & 530 & 0 & 0 & 0 & 27 & 26 & 1 \\
\hline Endosperm only & 5150 & 2421 & 2729 & 7954 & 2857 & 5097 & 1 & 0 & 1 & 842 & 462 & 380 \\
\hline Embryo only & 1 & 1 & 0 & 4272 & 1480 & 2792 & 0 & 0 & 0 & 619 & 607 & 12 \\
\hline
\end{tabular}

gene expression in the embryo than in the endosperm (> 10-fold difference), consistent with previous findings [22]. TaASN2 was the most highly expressed in both tissues, in both conditions ( $\mathrm{S}+$ and $\mathrm{S}-$ ), and at both time-points, accounting for several times the expression of all the others combined, again consistent with previous findings [22]. This was despite the apparent lack of expression of a $B$ genome TaASN2 homeologue. Some varieties lack a B genome TaASN2 homeologue [23], including Chinese Spring, which was the reference genome used in the study. Analysis of the RNA-seq reads did not reveal any single nucleotide polymorphisms to suggest that a $\mathrm{B}$ genome homeologue was being expressed; in other words, all the reads could be assigned to the $\mathrm{A}$ or $\mathrm{D}$ genome homeologues. We conclude that the $\mathrm{B}$ genome homeologue is absent in SR3 and Spark, as it is in Chinese Spring, or is present but not expressed. The data also showed for the first time the A genome homeologue of TaASN2 to be much more highly expressed (> 3-fold difference) than the $\mathrm{D}$ genome homeologue. This meant that the A genome homeologue of TaASN2 was responsible for more than half of the total asparagine synthetase gene expression in the grain under both treatments and at both timepoints.

There were also differences in expression of the three TaASN1 homeologues, with the B genome homeologue expressed at lower levels than the $\mathrm{A}$ and $\mathrm{D}$ genome homeologues. Of the two TaASN3 genes, TaASN3.1 was much more highly expressed than TaASN3.2, while TaASN4 expression was very low in all the samples.

There was approximately $80 \%$ more asparagine synthetase gene expression in the embryo of Spark compared with SR3 at $14 \mathrm{dpa}$, under both sulphur sufficiency and deficiency, almost entirely due to higher levels of TaASN2 expression in Spark compared with SR3 at this time-point (Fig. 4a). This could explain the higher concentration of free asparagine in Spark grain compared with SR3 [6]. The difference had reduced to approximately $15 \%$ by 21 dpa, under sulphur sufficiency (Fig. 4c), while under sulphur deficiency at this point expression was actually higher in SR3 than in Spark.

The effects of sulphur were complex and differed between the genotypes. There was no effect of sulphur in the embryo of either genotype at $14 \mathrm{dpa}$ (Fig. 4a), but at $21 \mathrm{dpa}$ the expression of the TaASN1 homeologue on chromosome $5 \mathrm{D}$ increased in response to sulphur deficiency in SR3 $(p=0.0478)$, as did the expression of both TaASN2 homeologues ( $p=$ 0.038 and 0.047 , for the homeologues on chromosomes $3 \mathrm{~A}$ and $3 \mathrm{D}$, respectively) (Fig. 4c). Clearly, such a response could explain the increased free asparagine accumulation observed in wheat grain in response to sulphur deficiency. However, the same response was 


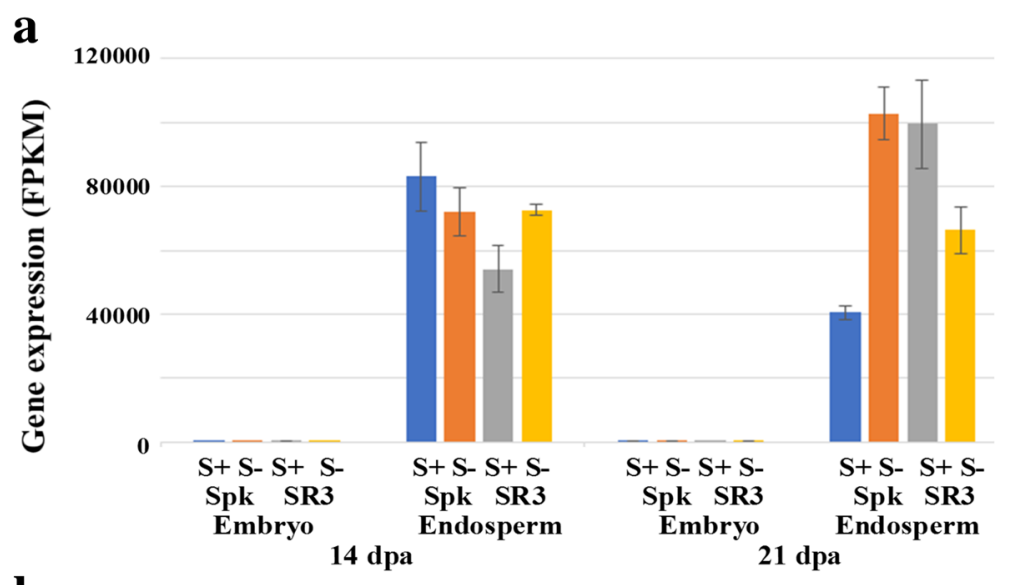

b

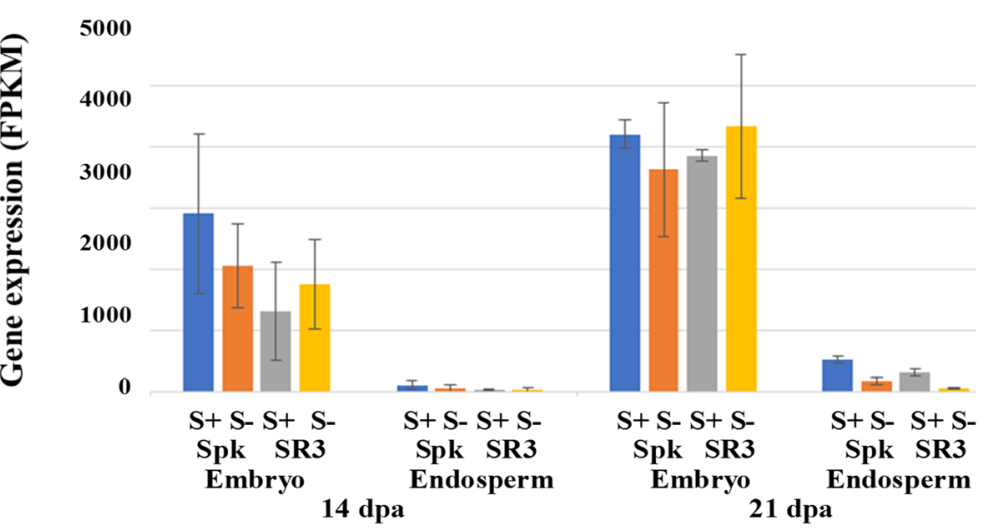

c

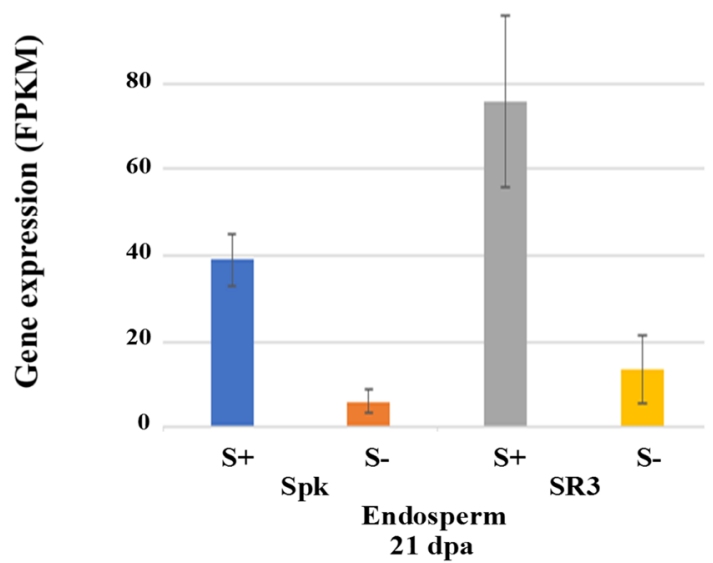

Fig. 3 Expression patterns of well-characterised genes in the embryo and endosperm of developing grain from two wheat (Triticum aestivum) genotypes, Spark and SR3, to validate the RNAseq dataset. The plants were grown with sulphur either supplied (S+) or withheld (S-) and sampled at 14 and 21 days post-anthesis (dpa). a Endosperm-specific expression of low molecular weight glutenin subunit gene. b Embryo-specific expression of late embryogenesis abundant (LEA12) gene. c Sulphur response of ethylene-insensitive 3-like 5 (EIL5) gene. Gene expression is represented in fragments per kilobase of transcript per million mapped reads (FPKM). Gene reference numbers are given in Additional file 2

not observed in Spark, in which both TaASN1 and TaASN2 expression decreased rather than increased in response to the low sulphur treatment. Given that the response seen in SR3 only occurred at the later timepoint of $21 \mathrm{dpa}$, one possible explanation is that
Spark was just behind SR3 developmentally and it was too early to see the response, but this is speculative.

In the endosperm at $14 \mathrm{dpa}$ (Fig. 4b), sulphur deficiency reduced expression of both TaASN1 and TaASN2 


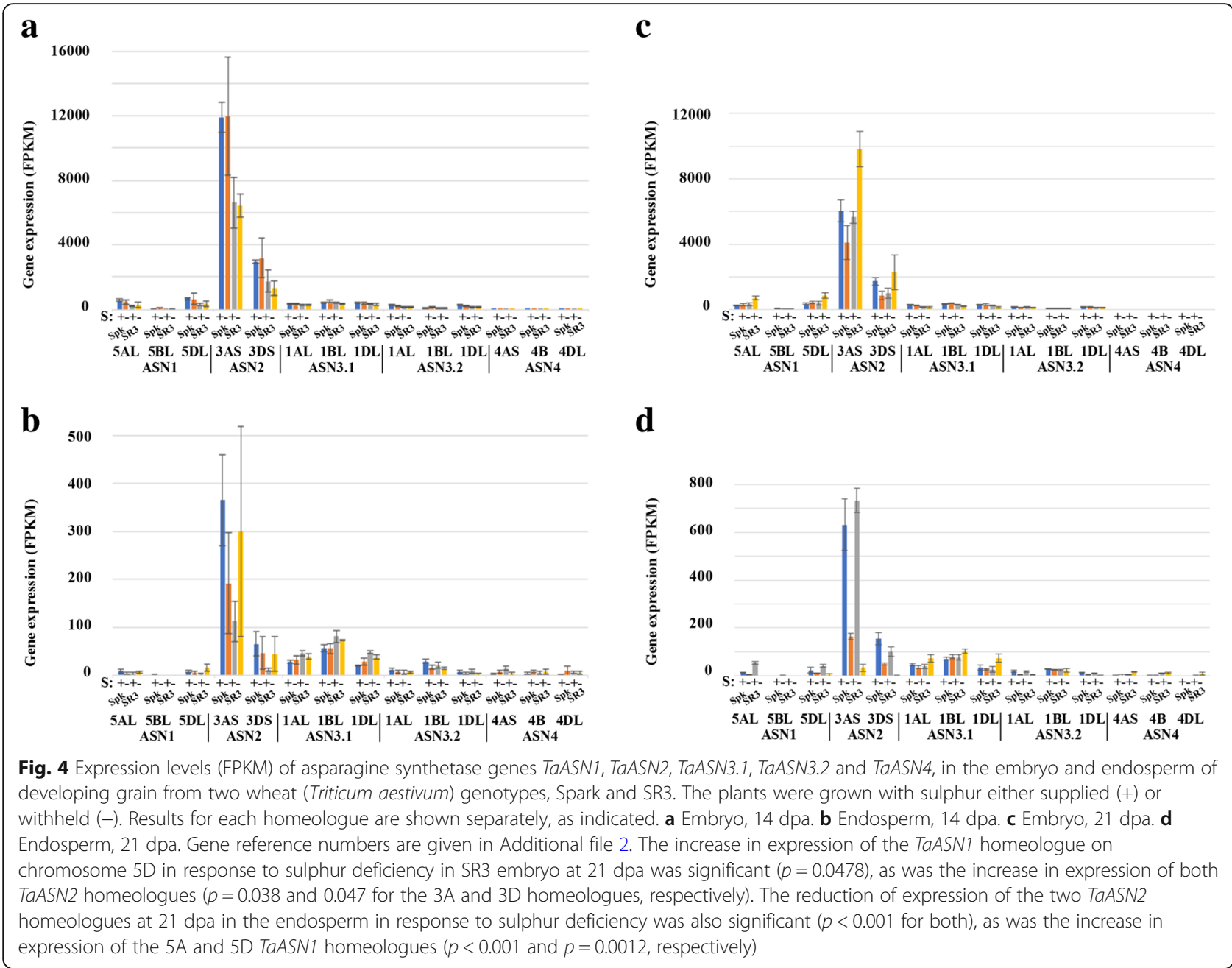

in Spark but increased it in SR3, although levels of expression were still only a fraction of those seen in the embryo. There was no great effect of sulphur deficiency on TaASN3 expression, and while TaASN4 expression increased in Spark but decreased in SR3, this was at comparatively very low levels of expression. At $21 \mathrm{dpa}$, sulphur deficiency reduced TaASN2 expression in both genotypes $(p<0.001$ for both homeologues $)$ but increased TaASN1 expression $(p<0.001$ and $p=0.0012$, respectively, for the homeologues on $5 \mathrm{~A}$ and $5 \mathrm{D}$; change not significant $(p>0.05)$ for the $5 \mathrm{~B}$ homeologue). TaASN1 therefore responded to sulphur in this tissue in the same way as in leaves. However, in contrast to the situation in leaves [22], its expression was dwarfed by that of TaASN2.

\section{Asparaginases}

Seven putative asparaginase genes were identified on each genome, based on the derived amino acid sequences of the encoded proteins (they are not annotated as asparaginases in the EnsemblPlants database). The reference numbers for the genes are given in Additional file 2. Six of the genes were located on chromosome $2 \mathrm{~A}$, with five homeologues in each case on $2 \mathrm{~B}$ and $2 \mathrm{D}$. Two unassigned sequences were probably the sixth $2 \mathrm{~B}$ and $2 \mathrm{D}$ homeologues. The other gene was located on chromosome 3, with homeologues on each of 3AS, 3B and 3DS. This gene encoded a protein with 96\% identity with an Aegilops tauschii asparaginase (accession number XP_020153640) and was of interest because all three homeologues showed significant $(p<0.01)$ increases in expression in response to sulphur deficiency at $21 \mathrm{dpa}$ in the endosperm of both Spark and SR3 (Fig. 5). All three homeologues were also expressed in the embryo, but the $\mathrm{B}$ genome version was expressed much more highly than the other two (Fig. 5). All three decreased in expression in the embryo in response to sulphur deficiency at $21 \mathrm{dpa}$ in SR3, the reduction in expression of the $\mathrm{B}$ genome homeologue being significant $(p=0.017)$. These responses meant that there was more asparaginase gene expression in the embryo than in the endosperm under sulphur sufficiency, but this was reversed under sulphur deficiency, particularly at $21 \mathrm{dpa}$. 


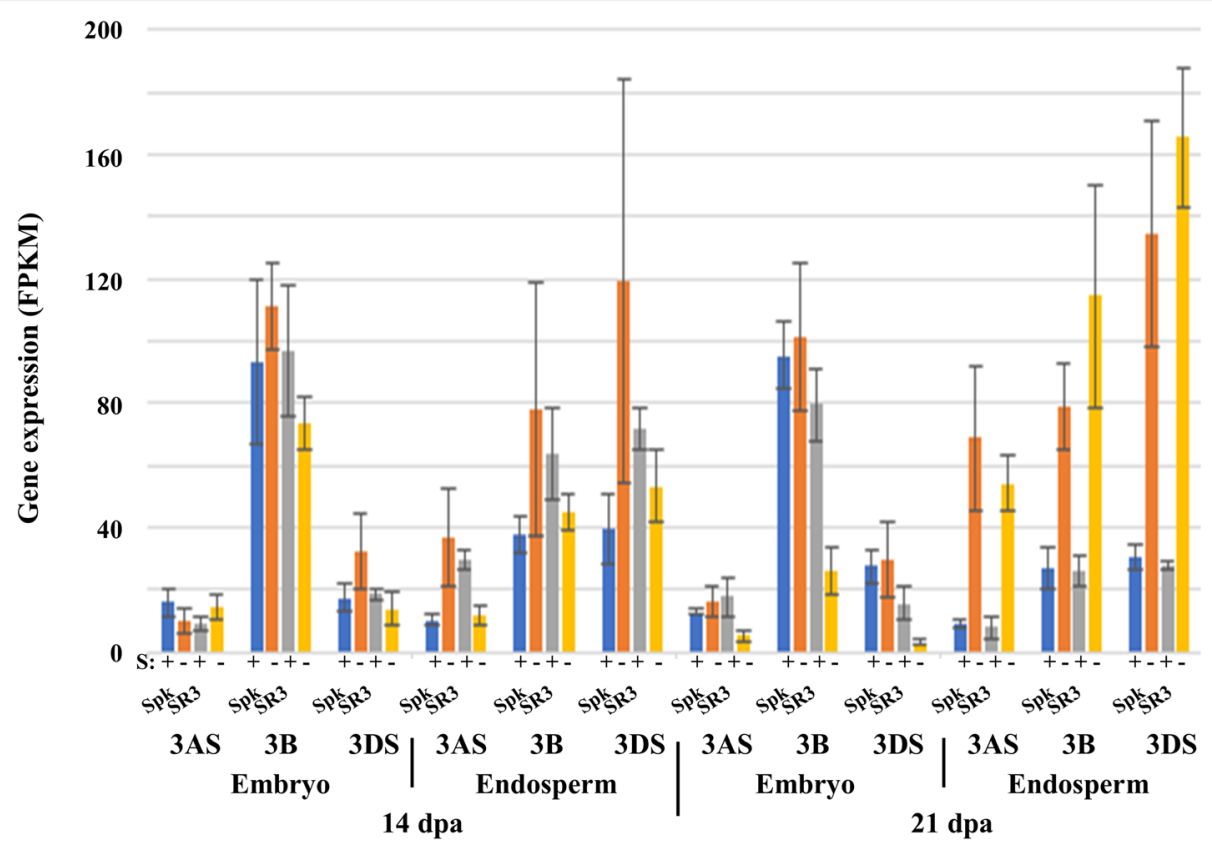

Fig. 5 Expression levels (FPKM) of asparaginase genes in the embryo and endosperm of developing grain from wheat (Triticum aestivum) genotypes Spark and SR3. Plants were grown with sulphur either supplied (+) or withheld (-) and analysed at 14 and 21 dpa. Results for each homeologue are shown separately, as indicated. Gene reference numbers are given in Additional file 2 . The increase in expression of all three homeologues in response to sulphur deficiency at $21 \mathrm{dpa}$ in the endosperm of both Spark and SR3 was significant $(p<0.01)$, as was the decrease in expression of the B genome homeologue in the embryo in response to sulphur deficiency at $21 \mathrm{dpa}$ in SR3 $(p=0.017)$

\section{Aspartate kinase}

Aspartate kinase has the potential to compete with asparagine synthetase for aspartate (Fig. 1). Genes encoding the enzyme were identified on chromosome 3 (3AL, 3B and 3DL), 4 (4AL only), and 5 (5BL and two homeologues on 5DL). Expression of these genes was generally higher in the embryo than the endosperm, with little change from 14 to $21 \mathrm{dpa}$ and little difference between the genotypes (Additional file 2: Figure S1). There were some responses to sulphur, notably of one of the 5DL homeologues in the endosperm at $21 \mathrm{dpa}$, but they were not significant $(p>$ $0.05)$. Overall the data did not suggest a major role for aspartate kinase in regulating free asparagine levels.

Enzymes of nitrogen assimilation: glutamine synthetase, glutamate synthase (GOGAT), nitrate reductase and nitrite reductase

Free asparagine becomes the most abundant free amino acid in wheat grain in response to sulphur deficiency, but there are also large increases in free glutamine and total free amino acid concentrations [4-7]. This makes enzymes of nitrogen assimilation of interest and the data showed that genes encoding nitrate reductase, nitrite reductase, glutamine synthetase and glutamate synthase (glutamine 2-oxyoglutarate aminotransferase; GOGAT) were all expressed.
The data for nitrate reductase and nitrite reductase are shown graphically in Fig. 6. The fact that these genes were expressed suggests strongly that nitrate was being transported into the grain, rather than nitrogen being imported entirely in the form of amino acids or other organic compounds. An NADPHdependent nitrate reductase-encoding gene was identified on chromosome 6, with homeologues on $6 \mathrm{AL}$, $6 \mathrm{BL}$ and $6 \mathrm{DL}$, all of which were expressed much more highly in the embryo than in the endosperm (Fig. 6a and b). Conversely, a gene encoding NADHdependent nitrate reductase was also identified on chromosome 6, with homeologues on 6AS, 6BS and $6 \mathrm{DS}$, and all of these were expressed much more highly in the endosperm than the embryo. There was a trend for expression of the gene encoding NADHdependent nitrate reductase to increase in response to sulphur deficiency in the endosperm at $21 \mathrm{dpa}$ but it was not significant $(p>0.05)$ (Fig. 6b). It was also notable that expression of the homeologues on chromosomes 6AS, 6DS, 6AL and 6BL was higher in Spark than SR3 at 14 dpa, with expression of the 6AS and 6DS homeologues continuing to be higher at 21 dpa. All three homeologues encoding the NADPHdependent nitrate reductase decreased in expression in the endosperm of SR3 in response to sulphur deficiency $(p<0.001)$, albeit from a low level. 


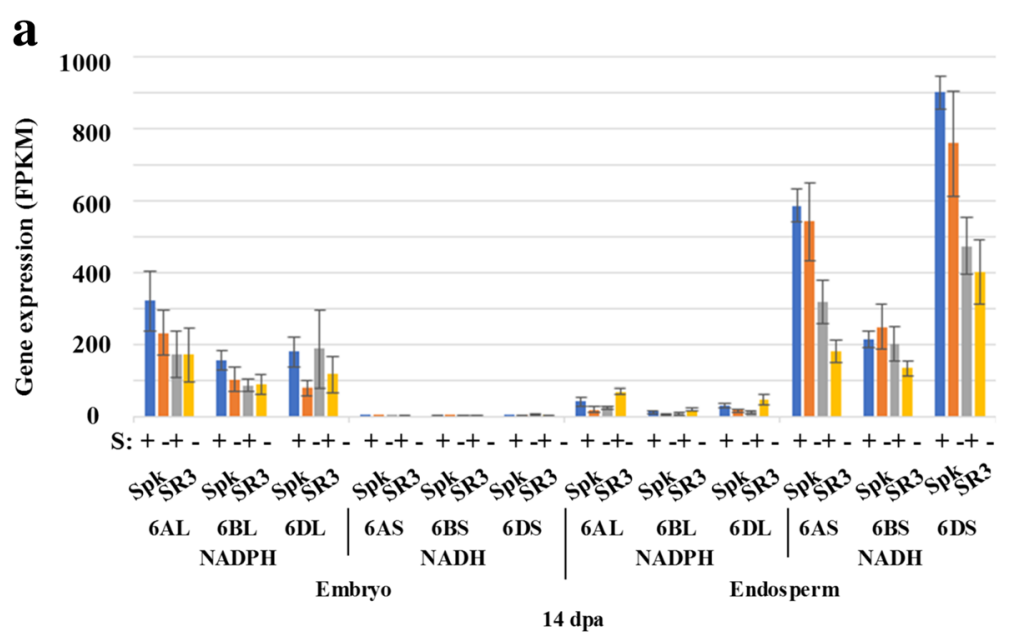

b

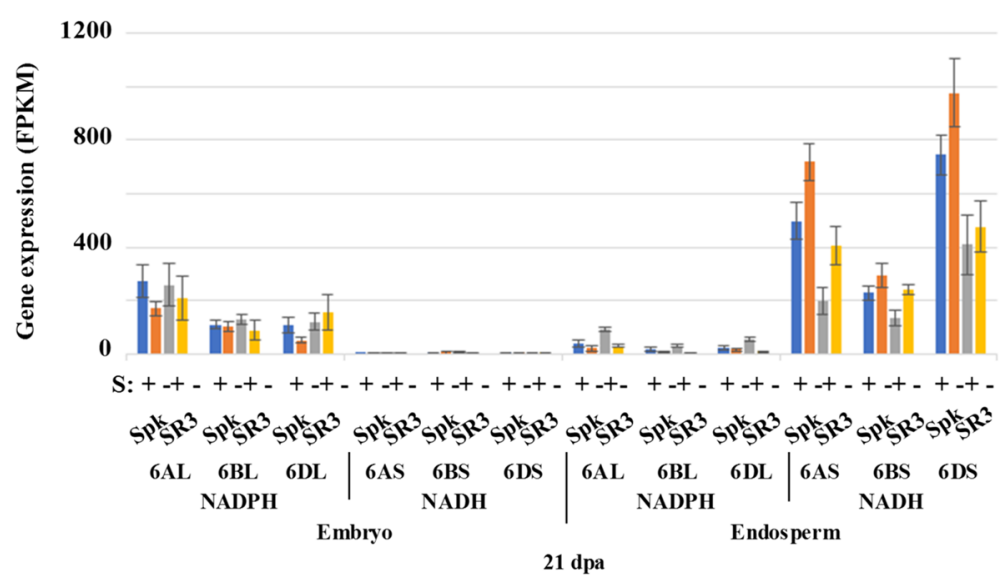

c

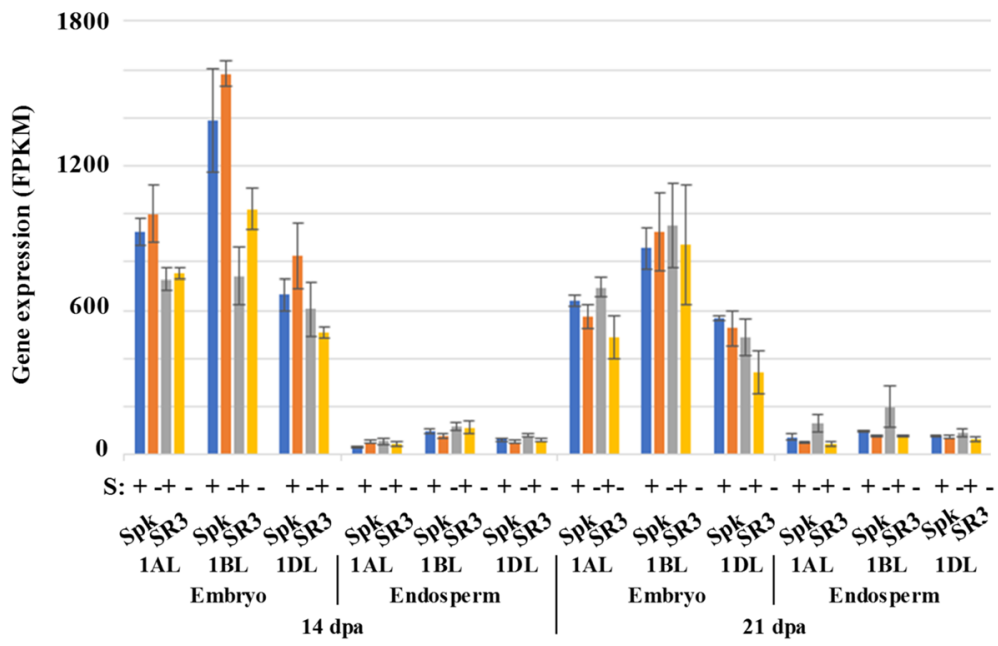

Fig. 6 Expression levels (FPKM) of nitrate and nitrite reductase genes in the embryo and endosperm of developing grain from wheat (Triticum aestivum) genotypes Spark and SR3. Plants were grown with sulphur either supplied (+) or withheld (-) and analysed at 14 and $21 \mathrm{dpa}$. a and $\mathbf{b}$ Genes encoding NADH- and NADPH-dependent nitrate reductase at 14 and $21 \mathrm{dpa}$, respectively. c Gene encoding nitrite reductase. Results for each homeologue are shown separately, as indicated. Gene reference numbers are given in Additional file 2 . The decrease in expression of the NADPH-dependent nitrate reductase gene in response to sulphur deficiency in the endosperm of SR3 at 21 dpa was significant $(p<0.001)$ 
A nitrite reductase gene was identified on chromosome 1 , with homeologues on $1 \mathrm{AL}, 1 \mathrm{BL}$ and $1 \mathrm{DL}$. These were expressed in both tissues but much more highly in the embryo than the endosperm (Fig. 6c). At 14 dpa, expression of the 1AL and 1BL homeologues in the embryo was much higher in Spark than SR3. A gene was also identified on chromosome 6, but this was expressed at much lower levels in both tissues.

Five genes encoding glutamine synthetase were identified, with GS1 (cytosolic) encoded by genes on chromosome 1, with homeologues on 1AL and 1BL (no 1D version), chromosome 4 (4AL, 4BS and 4DS) and chromosome 6 (6AL, 6BL and 6DL). GSr1 (cytosolic) was encoded by a gene on chromosome 4 (4AS, 4BL and 4DL), while GS2 (plastidic) was encoded by a gene on chromosome 2 (2AL, 2BL and 2DL). The genes encoding GSr1 and GS2 were expressed at relatively low levels (not surprisingly in the case of plastidic GS2) (Fig. 7). The GS1-encoding genes on chromosomes 1 and 6 were expressed much more highly in the embryo than the endosperm (Fig. 7a and c versus b and d), while the gene on chromosome 4 was expressed at high levels in both tissues. This meant that, overall, there was more expression of GS1 in the embryo than the endosperm. At 14 dpa under sulphur sufficiency, there was a trend for higher levels of expression in Spark than SR3 (Fig. 7a and b). This was most evident for the gene on chromosome 6 in the embryo and chromosome 4 in the endosperm. By 21 dpa this was reversed for some of the homeologues, and there were also contrasting responses to sulphur deficiency. There were large increases in expression of the 4AL and 6BL homeologues in the endosperm in SR3 at 14 dpa (Fig. 7b) ( $p=0.00113$ and 0.0299 , respectively), albeit that the $6 \mathrm{AL}$ homeologue was from a relatively low level. At $21 \mathrm{dpa}$ in the embryo there was no clear response to sulphur in

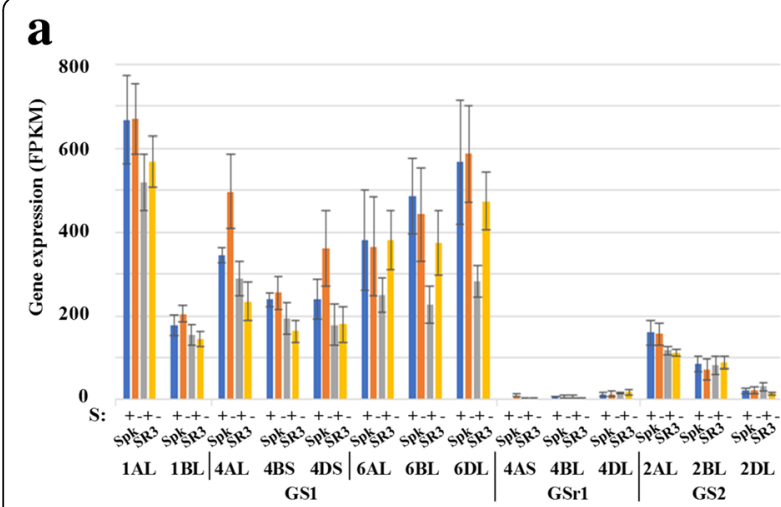

c

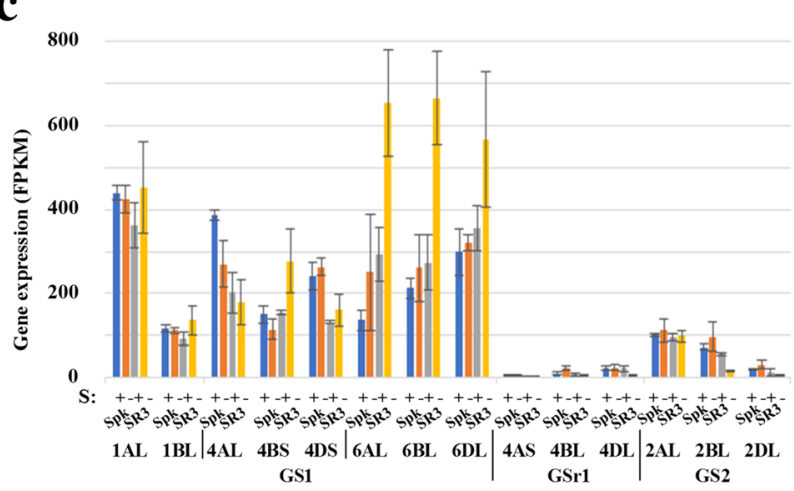

b

d

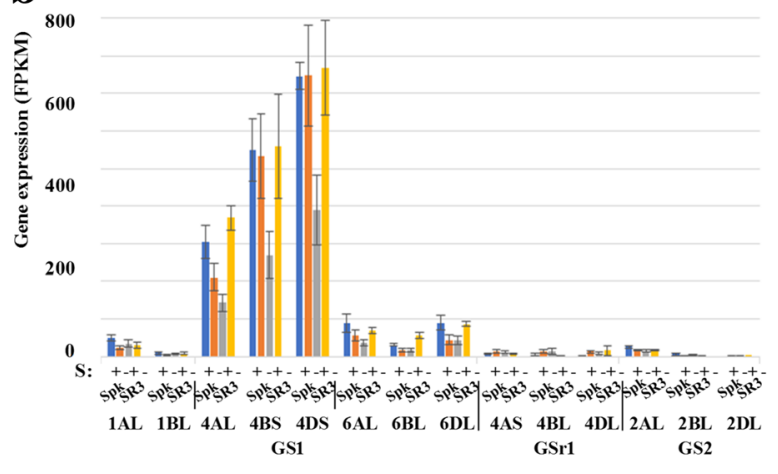

$\mathbf{d}_{1200}$

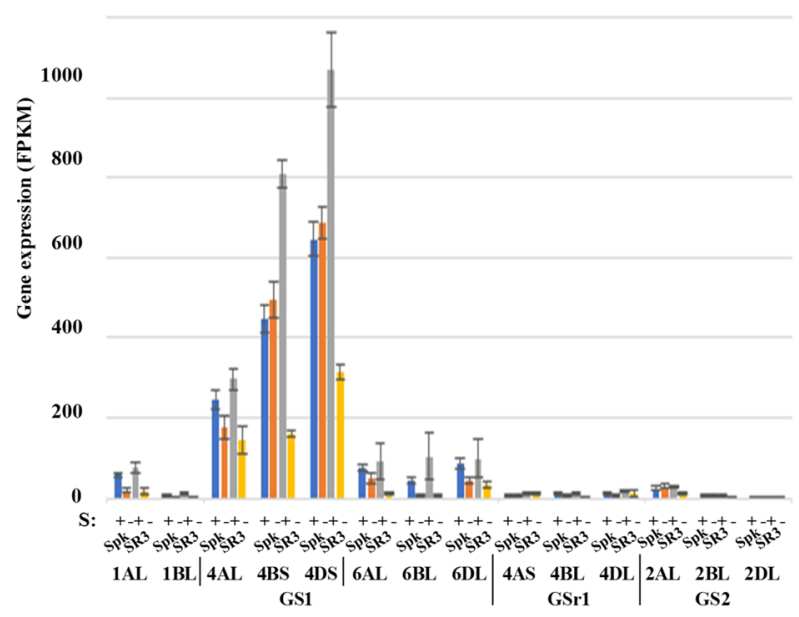

Fig. 7 Expression levels (FPKM) of genes encoding glutamine synthetases GS1, GSr1 and GS2 in the embryo and endosperm of developing grain from wheat (Triticum aestivum) genotypes Spark and SR3. The plants were grown with sulphur either supplied (+) or withheld (-). Results for each homeologue are shown separately, as indicated. a Embryo, 14 dpa. b Endosperm, 14 dpa. c Embryo, 21 dpa. d Endosperm, 21 dpa. Gene reference numbers are given in Additional file 2. The increase in expression of the $4 \mathrm{AL}$ and $6 \mathrm{BL}$ homeologues in the endosperm in SR3 at $14 \mathrm{dpa}$ in response to sulphur deficiency was significant ( $p=0.00113$ and 0.0299 , respectively), as was the increase in expression of the $6 \mathrm{AL}$ and $6 \mathrm{BL}$ homeologues in the embryo of SR3 at $21 \mathrm{dpa}$ ( $p=0.0401$ and 0.0164 , respectively). In the endosperm at $21 \mathrm{dpa}$, the decrease in expression of the $6 \mathrm{AL}, 6 \mathrm{BL}, 6 \mathrm{DL}, 4 \mathrm{DS}, 4 \mathrm{AL}$ and $1 \mathrm{AL}$ genes in $\mathrm{SR3}$ in response to sulphur deficiency was significant $(p<0.001$ for $1 \mathrm{AL}, 6 \mathrm{AL}, 4 \mathrm{BS}$ and $4 \mathrm{DS} ; p=$ 0.01961 for $6 \mathrm{BL}$ and 0.04599 for $6 \mathrm{DL}$ ) 
Spark (Fig. 7c), whereas in SR3 the expression of the 6AL and 6BL homeologues increased in response to sulphur deficiency $(p=0.0401$ and 0.0164 , respectively). In the endosperm at $21 \mathrm{dpa}$, on the other hand, expression of the 1AL, 4AL, 4DS, 6AL, 6BL and 6DL genes decreased in SR3 in response to sulphur deficiency (Fig. $7 \mathrm{~d})(p<0.001$ for 1AL, 4BS, 4DS and 6AL; $p=0.01961$ for 6BL and 0.04599 for $6 \mathrm{DL}$ ). Some genes showed the same response in Spark, but the highly expressed gene on chromosomes 4 did not. Yu et al. [17] also reported that GS gene expression was repressed by sulphur, but that study analysed expression at 7 dpa and only looked at the gene on chromosome 6 .

A single gene encoding glutamine oxoglutarate aminotransferase (GOGAT) was identified on chromosome 3 (3AL, 3BL and 3DL) (Additional file 2: Figure S2). The gene was expressed at much higher levels in the embryo than the endosperm. There was a trend for higher expression in the embryo under sulphur deficiency, but the differences were not significant $(p>0.05)$.

Sucrose nonfermenting-1-related protein kinase-1 (SnRK1) Sucrose nonfermenting-1-related protein kinase-1 (SnRK1) controls carbon metabolism through the modulation of enzyme activity and gene expression, including the re-allocation of carbon in response to a range of abiotic stresses (see $[26,27]$ for review). SnRK1 also promotes starch accumulation in potato tubers [28] and has been associated with the appearance of starch granules during rice endosperm development [29]. Its role in regulating asparagine synthetase gene expression has been demonstrated in Arabidopsis [30].

Cereals contain two types of gene encoding the catalytic subunit of SnRK1, called SnRK1a and SnRK1b, with SnRK1a being more similar to the dicot type and SnRK1b appearing to be monocot-specific and expressed at high levels in the developing endosperm [31-33]. The expression of the $S n R K 1$ genes in this study is shown in Fig. 8. A SnRK1a gene was identified on chromosome 1 (1AL, 1B and 1DL) and, as shown previously, was more highly expressed in the embryo than the endosperm (Fig. 8). A second SnRK1a gene was identified on chromosome 3 (3AL, 3BL and 3DL) but was expressed at much lower levels than the chromosome 1 gene, with both the $3 \mathrm{BL}$ and 3DL homeologues encoding proteins truncated by 150 amino acids at the C-terminal end. A SnRK1b gene was also located on chromosome 3 , but only $3 \mathrm{~B}$ and $3 \mathrm{DL}$, with no homeologue present on chromosome 3A. As expected, this gene showed much higher levels of expression in the endosperm than the embryo (Fig. 8). A second, truncated b-type gene was identified on chromosome 3B but was not expressed. A third type of SnRK1 gene was identified on chromosome 4, with two copies on chromosome 4A and one on each of 4BL and 4DL. Of these, one of the 4A homeologues and the 4D homeologue encoded full-length proteins and were expressed in both embryo and endosperm (Fig. 8). The 4B homeologue and the other $4 \mathrm{~A}$ homeologue were found to encode truncated proteins. Nevertheless, the 4B homeologue was expressed in similar fashion to the full-length 4A and 4D homeologues (Fig. 8). This gene type encoded a protein more similar to the SnRK1b type than the SnRK1a type, but since it showed a different expression pattern to the $S n R K 1 b$ gene (Fig. 8) it was called $S n R K 1 b^{*}$.

All three homeologues of the SnRK1a gene on chromosome 1 were more highly expressed in the embryo of Spark than SR3, particularly at $14 \mathrm{dpa}$, consistent with TaASN2 expression. There was no consistent response to sulphur at $14 \mathrm{dpa}$, but at $21 \mathrm{dpa}$ there was a trend for the $S n R K 1 b$ and $S n R K 1 b *$ genes to increase in expression in response to sulphur deprivation (Fig. 8c and d). This was significant for the $\operatorname{SnRK1b}$ homeologues on chromosomes 3B and 3DL in Spark endosperm $(p<0.01$ and $p<0.001$, respectively). The $3 \mathrm{~B}$ homeologue likewise showed a low level of expression in the embryo and this also rose significantly in Spark in response to sulphur deficiency $(p<$ 0.01 ). Expression of the 4BL and 4DL homeologues of the $S n R K 1 b^{*}$ gene rose significantly in response to sulphur deficiency in SR3 embryo ( $p=0.0426$ and 0.0377 , respectively), while expression of the 4DL homeologue also rose significantly in the endosperm of Spark $(p=0.0404)$. These data added to the evidence for a much stronger response to sulphur at the later timepoint and demonstrated a clear responsiveness of SnRK1 gene expression to sulphur, implicating this important metabolic regulator in the sulphur response.

\section{General control nonderepressible-2-type protein kinase (GCN2)}

Another protein kinase that has been shown to play a role in regulating asparagine synthetase gene expression in wheat is GCN2, which phosphorylates translation initiation factor eIF2 $\alpha$. GCN2 is encoded by a single gene in every plant species in which it has been identified and is the only plant eIF2 $\alpha$ kinase [34]. Its over-expression in transgenic wheat results in reduced total free amino acid and free asparagine concentration in the grain [35]. TaASN1 expression in the leaves of over-expressing plants is greatly reduced and does not increase in response to sulphur deficiency, whereas it does in wildtype wheat leaves [35].

Analysis of the dataset produced in this study confirmed that a single TaGCN2 gene was present on chromosome 2 (2AL, 2BL and 2DL), with the $2 \mathrm{BL}$ homeologue showing the highest expression level and the 2DL homeologue showing very low levels of expression (Fig. 9). Expression of all three homeologues increased in SR3 embryos at $21 \mathrm{dpa}$ under sulphur deficiency $(p=0.03114$ for the 2AL homeologue, $<0.01$ 


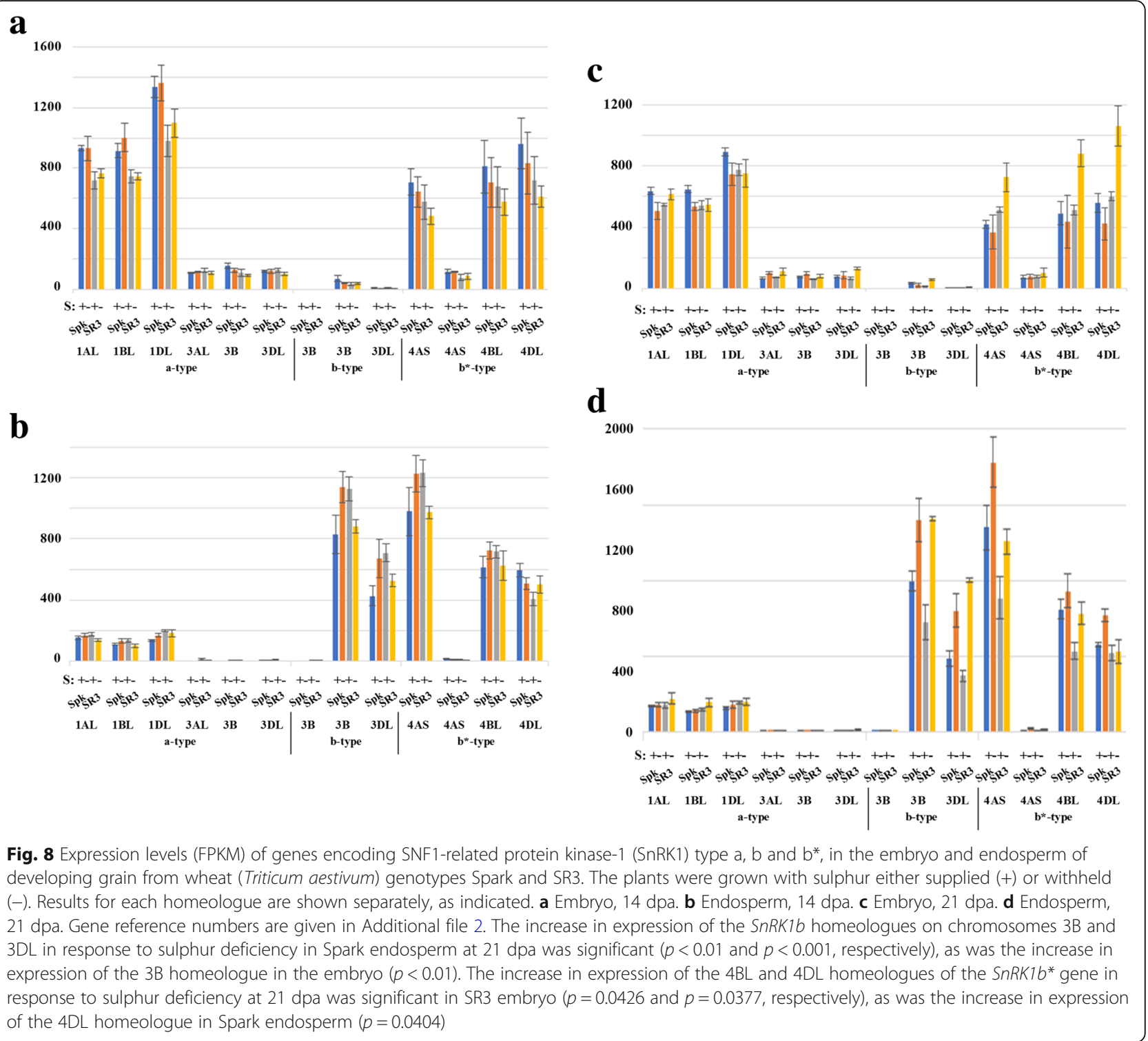

for the 2BL homeologue and 0.01313 for the 2DL homeologue). This can further explain the increased responsiveness of SR3 to sulphur.

\section{bZIP transcription factors}

A total of 576 transcription factors (inclusive of homeologues) showed changes in gene expression in response to sulphur deficiency (Additional file 2: Table S1). Basic Leucine Zipper Domain (bZIP) transcription factors were of interest because they are involved in regulating asparagine synthetase gene expression. For example, bZIPs regulate Arabidopsis AtASN1 gene expression via a bZIP binding site adjacent to the TATA box [30]. bZIPs are known to bind more than one target site, typically but not exclusively palindromic sequences with an ACGT core
[36], and the binding site in AtASN1 is a G-box (CACGTG). Transcription factors shown to bind this site in AtASN1 include bZIP11, which is involved in sucrose signalling and is regulated at the translational level [37]. However, the G-box of AtASN1 is not present in wheat asparagine synthetase gene promoters (Additional file 2: Figure S3a-c). The cDNA data that are provided for the TaASN2 genes in the EnsemblPlants database suggest that the TaASN2 mRNA has a long leader sequence, and the relatively short stretch of nucleotide sequence data upstream of the ATG translation start site available for the TaASN2 gene on chromosome 3AS from the database do not include an obvious TATA box. However, the longer nucleotide sequence data for the 3DS gene do contain a putative TATA sequence 625 base 


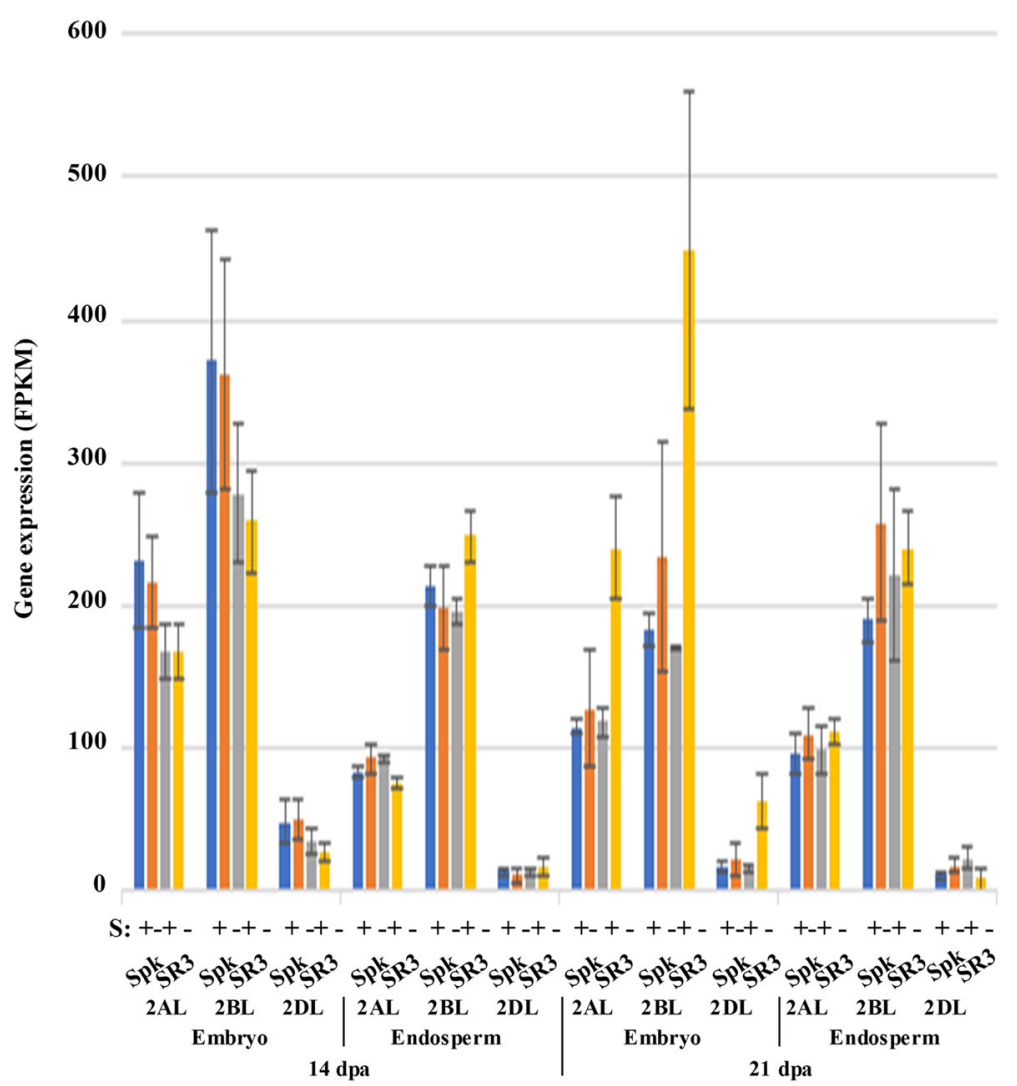

Fig. 9 Expression levels (FPKM) of genes encoding general control nonderepressible-2 (GCN2) protein kinase in the embryo and endosperm of developing grain from wheat (Triticum aestivum) genotypes Spark and SR3. Plants were grown with sulphur either supplied (+) or withheld (-) and analysed at 14 and $21 \mathrm{dpa}$. Results for each homeologue are shown separately, as indicated. Gene reference numbers are given in Additional file 2. The increase in expression of all three homeologues in response to sulphur deficiency in SR3 embryos at 21 dpa was significant ( $p=$ 0.03114 for the $2 \mathrm{AL}$ homeologue, $<0.01$ for the $2 \mathrm{BL}$ homeologue and 0.01313 for the 2DL homeologue)

pairs upstream of the ATG translation start site (Additional file 2: Figure S3d). Adjacent to this putative TATA box is a bZIP binding site, but it is a $\mathrm{C}$ box (GACGTC) rather than a G-box.

The TaASN1 gene contains a putative TATA box much closer to the ATG translation start site (Additional file 2: Figure S3a-c), but this does not have an adjacent bZIP binding site. However, a potential regulatory motif has previously been identified in TaASN1 genes [22] and this motif, ATGAGTCATC, is present in all three TaASN1 genes in the EnsemblPlants database (Additional file 2: Figure S3a-c). This motif is also present in cereal storage protein gene promoters, where it comprises the $\mathrm{N}$-motif and is responsible for positive and negative effects on gene expression in response to nitrogen availability [38]. It is identical to the binding site for GCN4 of budding yeast, a transcription factor that is regulated at the translational level as a result of GCN2 phosphorylation of translation initiation factor eIF2 $\alpha$ (see [34] for review). Paradoxically, plants do not have a direct homologue of GCN4, but the motif is recognised by several bZIP transcription factors, including Opaque2 of maize [39],
Opaque2 dimerising protein (OHP1/BLZ1) [40, 41] and SPA [42].

Genes encoding these transcription factors were identified in the dataset and a heatmap of their expression at $21 \mathrm{dpa}$, when some sulphur responses became evident, is shown in Fig. 10. A gene encoding an Opaque2-like transcription factor was identified on chromosome 7 (7AL, 7BL and 7DL), although it was annotated in the EnsemblPlants database simply as bZIP9. A gene on chromosome 6 (6AS, 6BS and 6DS) was also annotated as $b Z I P 9$, and although it encodes a transcription factor that is less similar to Opaque2, it was also included in the heatmap. A gene encoding the SPA transcription factor was identified on chromosome 1 (1AL, $1 \mathrm{BL}$ and 1DL), annotated as bZIP25, while a gene encoding an OHP1/BLZ1-like transcription factor was identified on chromosome 5 (5AL, 5BL and 5DL), annotated as bZIP63. Notably, bZIP9, bZIP25 and bZIP63 are implicated in the regulation of asparagine synthetase gene expression in Arabidopsis, along with bZIP11 and bZIP10 $[30,37]$, although their similarity to Opaque2, SPA and OHP1/BLZ1 has not been discussed previously to our 


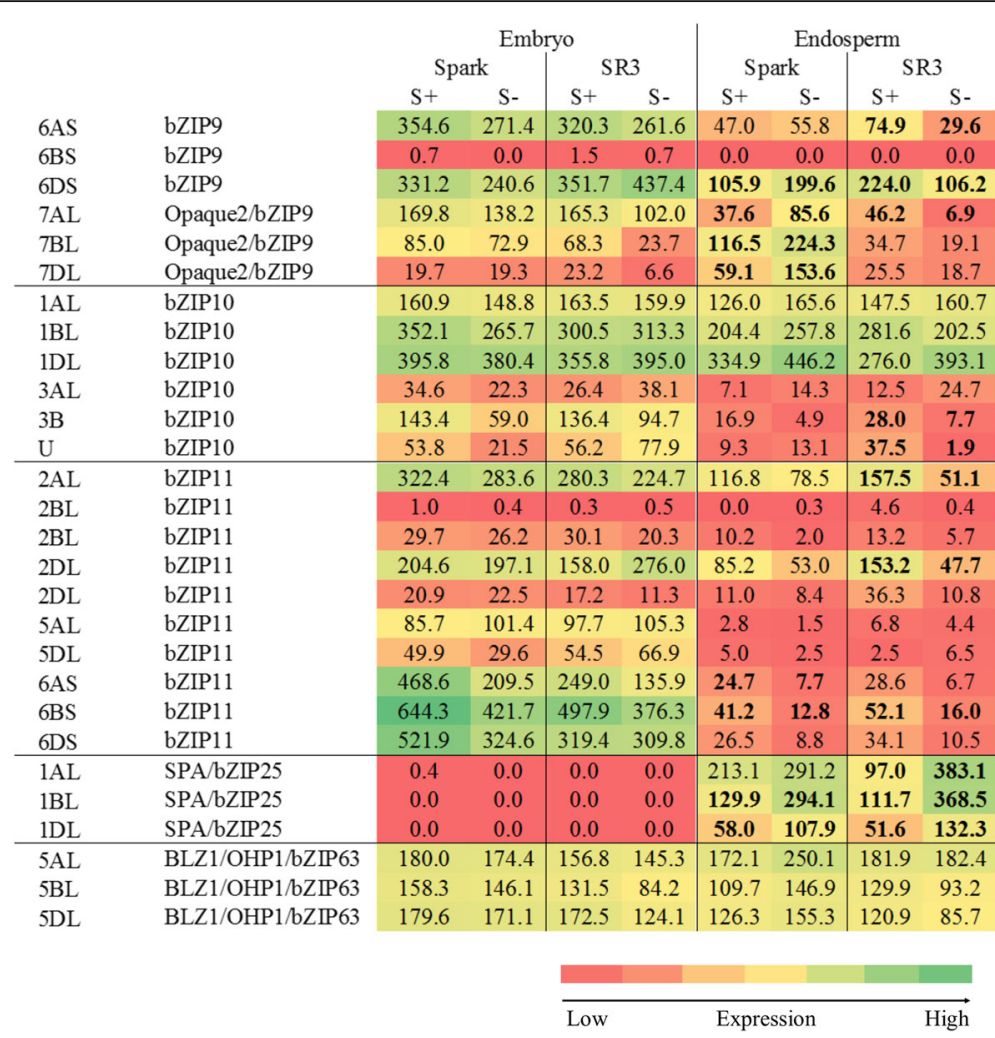

Fig. 10 Heatmap representing relative expression levels (FPKM) of genes encoding bZIP transcription factors bZIP9, Opaque2/bZIP9, bZIP10, bZIP11, SPA/bZIP25 and BLZ1/OHP1/bZIP63 classes in the embryo and endosperm of developing grain from wheat (Triticum aestivum) genotypes Spark and SR3 at $21 \mathrm{dpa}$. Plants were grown with sulphur either supplied (S+) or withheld (S-). Results for each homeologue are shown separately, as indicated. Gene reference numbers are given in Additional file 2. Significant $(p<0.05)$ changes in expression in response to sulphur are shown in bold

knowledge. Data on expression of bZIP10 genes (chromosomes 1 and 3) and bZIP11 genes (chromosomes 2, 5 and 6) are included in the heat map.

These transcription factors all have potential sites for phosphorylation by SnRK1, with the exception of bZIP11, which is translationally regulated [37]. SnRK1 preferentially phosphorylates a serine residue with a hydrophobic residue at +4 and -5 with respect to the serine and a basic residue at -3 , or less preferably at -4 [43]. The Opaque2/bZIP9 protein has such a sequence (Met-Lys-Lys-Cys-Ala-Ser-Glu-Leu-Glu-Leu) at its Nterminal end, with the target serine at position 6, while bZIP10 has the sequence Leu-Ala-Arg-Phe-Arg-SerAla-Ser-Gly-Ile with the target serine at position 26. The SPA/bZIP25 protein has the site Leu-Arg-Ile-Pro-PheSer-Gly-Ser-Pro-Leu with the target serine at position 233, while the OHP1/BLZ1/bZIP63 protein has two target sites in its $\mathrm{N}$-terminus (positions 6 and 39) and two overlapping sites in its C-terminal region (position 367 and 373) [44].

The heatmap (Fig. 10) shows differential expression between the homeologues and the genotypes for the Opaque2/bZIP9 gene on chromosome 7 , with the $7 \mathrm{~A}$ gene expressed more highly in the embryo than the endosperm but the other two being expressed more highly in the endosperm than the embryo in Spark but at similar levels in both tissues in SR3. Expression of all three homeologues increased significantly in response to sulphur deprivation in the endosperm of Spark $(p<0.01$ for the 7AL homeologue; $p=0.0398$ and 0.0405 , respectively, for the 7BL and 7DL homeologues), but the 7A gene decreased in expression in SR3 endosperm in response to sulphur deficiency $(p<0.001)$ while the other two homeologues showed no significant change $(p>$ $0.05)$. The $6 \mathrm{~A}$ and $6 \mathrm{D}$ homeologues of the other bZIP9 gene showed similar expression patterns, while the $6 \mathrm{~B}$ homeologue was hardly expressed at all.

The bZIP10 gene on chromosome 3 was expressed at low levels in both tissues, but the 3B homeologue and an unassigned gene that was almost certainly the 3D homeologue did show a significant reduction in expression $(p=$ 0.367 and $p<0.001$, respectively, for the 3B and 3D homeologues), albeit from already low levels, in response to sulphur deficiency in SR3. The bZIP10 gene on chromosome 1 , on the other hand, was expressed at relatively high levels in both tissues but showed no response to sulphur. 
The bZIP11 genes on chromosome 2 showed differential expression between homeologues, with both genes on chromosome $2 \mathrm{~B}$ and one of the genes on 2D expressed at low levels, but the other $2 \mathrm{D}$ gene and the only gene on chromosome $2 \mathrm{~A}$ expressed at relatively high levels particularly in the embryo. These two genes also showed reduced expression in the endosperm in response to sulphur deficiency, but this was significant only in SR3 $(p<0.001$ for the 2AL and 2DL homeologues). Neither homeologue of the bZIP11 gene on chromosome 5 was highly expressed, but both (5AL and 5DL) were expressed at higher levels in the embryo than the endosperm. The most highly expressed of the bZIP11 genes was the one on chromosome 6 (6AS, 6BS and 6DS). As with the other bZIP11 genes, there was higher expression in the embryo than the endosperm, and there was also a trend for lower expression in the endosperm in response to sulphur deficiency. This was significant $(p=0.0419)$ for the 6AS homeologue in Spark and for the 6BS homeologue in both genotypes $(p=$ 0.0229 for Spark and 0.03903 for SR3).

The SPA/bZIP25 gene on chromosome 1 was expressed in the endosperm but not at all in the embryo. Expression of all three homeologues increased significantly at $21 \mathrm{dpa}$ in SR3 endosperm in response to sulphur deficiency $(p<0.01)$, while the $1 \mathrm{BL}$ and 1DL homeologues also increased in Spark ( $p=0.03$ for both). The endosperm-specific expression of this transcription factor would be consistent with a role in regulating storage protein gene expression but suggests that it is not involved in regulating asparagine synthetase gene expression.

The OHP1/BLZ1/bZIP63 transcription factor gene on chromosome 5 , on the other hand, was expressed at similar levels in both endosperm and embryo, and clearly its expression in the embryo suggests that regulating storage protein gene expression is not its only role. It showed no response to sulphur, but as with all of the transcription factors its primary regulation may be post-transcriptional; indeed, this seems likely given its multiple SnRK1 target sites.

\section{Discussion}

The first observation to be made on the data is that there were clear differences between the genotypes with respect to the expression of key genes involved in asparagine metabolism. Secondly, sulphur responses were much more evident at $21 \mathrm{dpa}$ than $14 \mathrm{dpa}$, and much more evident in SR3 than Spark. Both genotypes have been shown previously to respond to sulphur deficiency with a massive accumulation of free asparagine in the grain [6], so this was unexpected. It is possible that Spark was simply behind SR3 developmentally and 21 dpa was too early to see the $\mathrm{S}$ response in this genotype.
The study confirmed TaASN2 to be the most highly expressed asparagine synthetase gene in the grain, with expression in the embryo much higher than in the endosperm. Indeed, the data suggest strongly that the embryo is the organ in which grain asparagine levels are determined. An obvious possible explanation of why Spark accumulates more free asparagine in the grain than SR3 was provided by the higher expression of TaASN2 in Spark during early development (14 dpa), and there was also a trend for genes encoding enzymes of nitrogen assimilation to be more highly expressed in Spark than SR3 when sulphur was supplied, consistent with the higher levels of total free amino acids in the grain of that genotype. Interestingly, genes encoding both nitrate reductase and nitrite reductase were expressed, suggesting that nitrate must be imported into the grain.

TaASN2 and glutamine synthetase gene expression in the embryo of SR3 increased in response to sulphur deficiency at $21 \mathrm{dpa}$, while asparaginase gene expression decreased. Asparagine synthetase and asparaginase gene expression in the endosperm responded in the opposite way, falling and rising, respectively, in response to sulphur deficiency. This suggests that the asparagine that accumulates in the endosperm in response to sulphur deficiency is imported from the embryo or elsewhere. We speculate that asparaginase is expressed in readiness to remobilise the free asparagine if sulphur becomes available, for example if the roots reach a source of sulphur in the soil, or at germination. This would mean that the enzyme would have to be inactive until required.

Another notable observation was the expression of regulatory protein kinases, SnRK1 and GCN2. Both have been implicated in regulating asparagine synthetase gene expression before [30,35], and both showed responses to sulphur deficiency that would be consistent with that role. bZIP transcription factors, including four with SnRK1 target sites, were also expressed, and putative regulatory motifs at which these transcription factors could bind were identified in both TaASN1 and TaASN2 promoters, including the $\mathrm{N}$-motif identified previously in TaASN1 [22]. Three of these transcription factors, Opaque2/bZIP9, SPA/bZIP25 and BLZ1/OHP1/bZIP63, are known to bind the $\mathrm{N}$-motif. The data are certainly consistent with SnRK1 regulating TaASN1 and TaASN2 expression through these transcription factors.

Another general observation from the data was that the homeologues of many genes showed differential expression patterns and responses. In the context of the aims of the study, this was most important for TaASN2, the chromosome 3A homeologue of which was expressed at much higher levels than the 3D homeologue, while the $3 \mathrm{~B}$ homeologue was either not expressed or was missing altogether. 


\section{Conclusions}

The study provided extensive new data on the genetic control of free asparagine accumulation in wheat grain and its response to sulphur supply. It showed the embryo to be the organ in which grain asparagine levels are determined, based on the levels of expression of the key genes involved, notably those encoding asparagine synthetase, and identified genes encoding signalling and metabolic proteins involved in asparagine metabolism that respond to sulphur availability. Asparagine synthetase gene TaASN2 was confirmed as a logical target for genetic interventions aimed at reducing the asparagine content of wheat and other cereal grains, and the data suggested that interventions aimed at the A genome homeologue alone could be effective. The study also identified genes encoding other metabolic enzymes and signalling factors that could be targeted.

\section{Methods}

\section{Sample preparation}

Wheat plants of variety Spark and doubled haploid line SR3 from a Spark $\times$ Rialto mapping population [16] were grown from seed held at Rothamsted Research by Tanya Curtis and Nigel Halford. The seed were derived from lines originally supplied by the John Innes Centre Wheat Genetics Group, Norwich, UK. The plants were grown in vermiculite in a glasshouse with a $16 \mathrm{~h}$ day-length (supplemental lighting was used as necessary) and a minimum temperature of $16^{\circ} \mathrm{C}$. Vermiculite does not retain nutrients, so the only nutrition available to the plants came from liquid feed solution. Feeding was started 3 weeks after potting and continued every 2 days until harvest. Plants were supplied with either a medium containing a full nutrient complement of potassium, phosphate, calcium, magnesium, sodium, iron, nitrate $\left(2 \mathrm{mM} \mathrm{Ca}\left(\mathrm{NO}_{3}\right)_{2}\right.$ and $\left.1.6 \mathrm{mM} \mathrm{Mg}\left(\mathrm{NO}_{3}\right)_{2}\right)$ and sulphate ions (1.1 mM $\left.\mathrm{MgSO}_{4}\right)[4,6]$, or the same medium containing one tenth the concentration of $\mathrm{MgSO}_{4}$. Distilled water was supplied as required to prevent water stress. A randomised design was used for the pots in the glasshouse.

Ears were tagged at anthesis, grain sampled at 14 and $21 \mathrm{dpa}$, and caryopses dissected under a microscope to isolate the embryo and endosperm. Dissected samples were immediately frozen in liquid nitrogen and stored at $-80^{\circ} \mathrm{C}$. There were four biological replicates for each of the two time-points, two treatments and two varieties, making a total of 32 embryo and 32 endosperm samples.

\section{RNA extraction and RNA-seq analysis}

The RNA extraction method was modified from Chang et al. [45]. Frozen tissues were ground in liquid nitrogen and extracted in CTAB buffer (2\% (w/v) cetyl trimethylammonium bromide, $2 \%(\mathrm{w} / \mathrm{v})$ polyvinylpyrrolidone (PVP) K 30, $100 \mathrm{mM}$ Tris- $\mathrm{HCl}, \mathrm{pH} 8.0,25 \mathrm{mM}$ ethylenediaminetetraacetic acid (EDTA), 2.0 M NaCI, $0.5 \mathrm{~g} / \mathrm{L}$ spermidine, 2\% $(w / v) \beta$-mercaptoethanol). The supernatant was extracted twice with chloroform: isoamyl alcohol (IAA) (24:1) to remove proteins. RNA was precipitated by addition of 0.25 vol. of $10 \mathrm{M} \mathrm{LiCl}$ and incubation on ice overnight. The RNA pellet was dissolved in SSTE buffer $(1.0 \mathrm{M} \mathrm{NaCl}$, 0.5\% (w/v) SDS, $10 \mathrm{mM}$ Tris $\mathrm{HCl} \mathrm{pH} 8.0,1 \mathrm{mM}$ EDTA) to remove polysaccharides and extracted once with chloroform: isoamyl alcohol. After ethanol precipitation, total RNA was dissolved in diethyl pyrocarbonate-treated water and stored at $-80^{\circ} \mathrm{C}$. Total RNA was treated with deoxyribonuclease and purified through RNeasy mini spin columns (Qiagen, Crawley, UK).

The RNA-seq analysis was performed by GATC Biotech (Konstanz, Germany) using the NGSelect option, generating 15 million $125 \mathrm{bp}$ paired-end strand-specific reads per sample.

\section{Bioinformatics}

The reads were not trimmed. HISAT2 (v2.0.5) was used to map the RNA-seq data to the most comprehensive available Triticum aestivum cv. Chinese Spring reference at the time of analysis (TGACv1) with FeatureCounts (v1.5.1) used to count against the reference annotation exons using the strand-specific option. Mapping rates across samples for SR3 were: 1,364,906,937 in total; 1, $225,035,426$ properly paired $(89.75 \%) ; 50,200,825$ singletons $(3.68 \%) ; 22,083,695$ with mate mapped to a different chromosome $(\mathrm{mapQ}>=5)$. For Spark the mapping rates were: $1,514,802,531$ in total; $1,349,695,534$ properly paired (89.10\%); 62,138,374 singletons (4.10\%); 29,187, 503 with mate mapped to a different chromosome (mapQ $>=5)$. The $\mathrm{R}$ package DeSeq2 (v1.22.2) was used for differential expression and genes functionally annotated using Blast2Go (v4.2). Genes with an adjusted $p$ value of $<0.05$ were regarded as differentially expressed.

Gene Ontology enrichment analysis was performed using the Fisher's Exact Test in Blast2GO version 5.2.5 [46]. The enrichment analysis was limited to Molecular Function and Biological Process categories and the FDR threshold was set to 0.05 . The results were summarised further to the most specific GO term.

\section{Additional files}

Additional file 1: Results of functional enrichment analysis. (XLSX $336 \mathrm{~kb}$ )
Additional file 2: EnsembIPlants reference numbers for genes
discussed in the paper. Figure S1. Expression levels (FPKM) of genes
encoding aspartate kinase. Figure S2. Expression levels (FPKM) of genes
encoding glutamate synthase (GOGAT). Figure S3. Asparagine synthetase
gene promoter nucleotide sequences. Table S1. Transcription factors
differentially expressed in response to sulphur deficiency. (DOCX $1653 \mathrm{~kb}$ )

Abbreviations

bZIP: Basic leucine zipper; EFSA: European Food Safety Authority; FPKM: Fragments per kilobase of transcript per million mapped reads; GCN2: General control nonderepressible-2; GOGAT: Glutamate synthase 
(glutamine 2-oxyoglutarate aminotransferase); RNA-seq: RNA sequencing; SNF1: Sucrose nonfermenting-1; SnRK1: SNF1-related protein kinase-1

\section{Acknowledgements}

Not applicable.

\section{Authors' contributions}

TYC performed most of the experimental work, including the plant growth, treatment and sample preparation. YW prepared the RNA samples. RK and AG-U performed the bioinformatics and statistical analyses of the data. SR analysed the dataset, identified genes of interest and produced the graphs and heatmap used in the figures. NGH was the project leader and prepared the manuscript. All authors read and approved the final manuscript.

\section{Funding}

TC: BBSRC stand-alone LINK project BB/I020918/1. SR: BBSRC SWBio iCASE DTP Studentship. NGH, RK and AGU were supported at Rothamsted Research by the BBSRC via the Designing Future Wheat Programme (BB/P016855/1). $B B S R C$ had no role in the design of the study, collection, analysis or interpretation of the data, or in the writing of the manuscript.

\section{Availability of data and materials}

The raw data has been deposited in European Nucleotide Archive (ENA) and is publicly available (https://www.ebi.ac.uk/ena) using the study accession: PRJEB31122

\section{Ethics approval and consent to participate} Not applicable.

\section{Consent for publication}

Not applicable.

\section{Competing interests}

TC was supported by the Biotechnology and Biological Sciences Research Council (BBSRC) of the UK and a consortium of companies and organisations through stand-alone LINK project BB/I020918/1. SR was supported by a BBSRC SWBio iCASE DTP Studentship, with partners: Keith Edwards (University of Bristol), AHDB, KWS UK Ltd., Saaten Union UK Ltd., RAGT Seeds Ltd., Syngenta UK Ltd., and Limagrain UK Ltd. NGH, RK and AGU were supported at Rothamsted Research by the BBSRC via the Designing Future Wheat Programme (BB/P016855/1).

\section{Author details}

${ }^{1}$ Plant Sciences Department, Rothamsted Research, Harpenden, Hertfordshire AL5 2JQ, UK. ${ }^{2}$ Computational and Analytical Sciences Department, Rothamsted Research, Harpenden, Hertfordshire AL5 2JQ, UK. ${ }^{3}$ Present Address: Curtis Analytics Ltd, Daniel Hall Building, Rothamsted RoCRE, Harpenden AL5 2JQ, UK. ${ }^{4}$ Present Address: The European Bioinformatics Institute (EMBL-EBI), Wellcome Genome Campus, Hinxton, Cambridgeshire CB10 1SD, UK.

Received: 1 May 2019 Accepted: 23 July 2019

Published online: 01 August 2019

\section{References}

1. Mottram DS, Wedzicha BL, Dodson AT. Acrylamide is formed in the Maillard reaction. Nature. 2002;419:448-9.

2. Stadler RH, Blank I, Varga N, Robert F, Hau J, Guy PA, Robert MC, Riediker S. Acrylamide from Maillard reaction products. Nature. 2002;419:449-50.

3. Zyzak DV, Sanders RA, Stojanovic M, Tallmadge DH, Eberhart BL, Ewald DK, Gruber DC, Morsch TR, Strothers MA, Rizzi GP, Villagran MD. Acrylamide formation mechanism in heated foods. J Agric Food Chem. 2003;51:4782-7.

4. Muttucumaru N, Halford NG, Elmore JS, Dodson AT, Parry M, Shewry PR, Mottram DS. The formation of high levels of acrylamide during the processing of flour derived from sulfate-deprived wheat. J Agric Food Chem. 2006;54:8951-5.

5. Granvogl M, Wieser $H$, Koehler P, von Tucher S, Schieberle P. Influence of sulphur fertilization on the amounts of free amino acids in wheat. Correlation with baking properties as well as with 3aminopropionamide and acrylamide generation during baking. J Agric Food Chem. 2007:55:4271-7.
6. Curtis TY, Muttucumaru N, Shewry PR, Parry MA, Powers SJ, Elmore JS, Mottram DS, Hook S, Halford NG. Evidence for genetic and environmental effects on free amino acid levels in wheat grain: implications for acrylamide formation during processing. J Agric Food Chem. 2009;57:1013-21.

7. Curtis TY, Powers SJ, Balagiannis D, Elmore JS, Mottram DS, Parry MAJ, Raksegi M, Bedő Z, Shewry PR, Halford NG. Free amino acids and sugars in rye grain: implications for acrylamide formation. J Agric Food Chem. 2010; 58:1959-69.

8. Postles J, Powers SJ, Elmore JS, Mottram DS, Halford NG. Effects of variety and nutrient availability on the acrylamide forming potential of rye grain. J Cereal Sci. 2013:57:463-70.

9. International Agency for Research on Cancer. Some industrial chemicals; IARC monographs on the evaluation of carcinogenic risks to humans, vol. 60. Lyon: International Agency for Research on Cancer; 1994.

10. EFSA Panel on Contaminants in the Food Chain (CONTAM). Scientific opinion on acrylamide in food. EFSA J. 2015;13:4104.

11. European Commission. Commission Regulation EU. 2017/2158 establishing mitigation measures and benchmark levels for the reduction of the presence of acrylamide in food. Brussels: European Commission; 2017.

12. Curtis TY, Bo V, Tucker A, Halford NG. Construction of a network describing asparagine metabolism in plants and its application to the identification of genes affecting asparagine metabolism in wheat under drought and nutritional stress. Food Energy Secur. 2018;7:e00126.

13. Lea PJ, Sodek L, Parry MA, Shewry PR, Halford NG. Asparagine in plants. Ann Appl Biol. 2007;150:1-26.

14. Curtis TY, Powers SJ, Halford NG. Effects of fungicide treatment on free amino acid concentration and acrylamide-forming potential in wheat. J Agric Food Chem. 2016;64:9689-96.

15. Curtis TY, Powers SJ, Wang R, Halford NG. Effects of variety, year of cultivation and sulphur supply on the accumulation of free asparagine in the grain of commercial wheat varieties. Food Chem. 2018;239:304-13.

16. Snape JW, Foulkes MJ, Simmonds J, Leverington M, Fish $\sqcup$, Wang $Y$, Ciavarrella M. Dissecting gene $\times$ environmental effects on wheat yields via QTL and physiological analysis. Euphytica. 2006;154:401-8.

17. Yu Z, Juhasz A, Islam S, Diepeveen D, Zhang J, Wang P, Ma W. Impact of mid-season sulphur deficiency on wheat nitrogen metabolism and biosynthesis of grain protein. Sci Rep. 2018;8:2499.

18. Shewry PR, Halford NG, Lafiandra D. The genetics of wheat gluten proteins. Adv Genet. 2003;49:111-84.

19. Wise MJ, Tunnacliffe A. POPP the question: what do LEA proteins do? Trends Plant Sci. 2004;9:13-7.

20. Lu C, Howarth JR, Barraclough PB, Edwards KJ, Hawkesford MJ. Transcriptome analysis of wheat responses to sulfur nutrition. In: Saito K, De Kok LJ, Stulen I, Hawkesford MJ, Schnug E, Sirko A, Rennenberg H, editors. Sulfur transport and assimilation in plants in the post genomic era. Leiden: Backhuys Publishers; 2005. ISBN 9057821664.

21. Maruyama-Nakashita A, Nakamura Y, Tohge T, Saito K, Takahashia H. Arabidopsis SLIM1 is a central transcriptional regulator of plant sulfur response and metabolism. Plant Cell. 2006;18:3235-51.

22. Gao R, Curtis TY, Powers SJ, Xu H, Huang J, Halford NG. Food safety: structure and expression of the asparagine synthetase gene family of wheat. J Cereal Sci. 2016;68:122-31.

23. Xu H, Curtis TY, Powers SJ, Raffan S, Gao R, Huang J, Heiner M, Gilbert D, Halford NG. Genomic, biochemical and modelling analyses of asparagine synthetases from wheat. Front Plant Sci. 2018;8:2237.

24. Curci PL, Bergès $H$, Marande W, Maccaferri M, Tuberosa R, Sonnante G. Asparagine synthetase genes (AsnS1 and AsnS2) in durum wheat: structural analysis and expression under nitrogen stress. Euphytica. 2018;214:36.

25. Wang H, Liu D, Sun J, Zhang A. Asparagine synthetase gene TaASN1 from wheat is up-regulated by salt stress, osmotic stress and ABA. J Plant Physiol. 2005;162:81-9.

26. Hey SJ, Byrne E, Halford NG. The interface between metabolic and stress signalling. Ann Bot. 2010;105:197-203.

27. Paul MJ, Gonzalez-Uriarte A, Griffiths CA, Hassani-Pak K. The role of trehalose 6-phosphate in crop yield and resilience. Plant Physiol. 2018;177:12-23.

28. McKibbin RS, Muttucumaru N, Paul MJ, Powers SJ, Burrell MM, Coates S, Purcell PC, Tiessen A, Geigenberger P, Halford NG. Production of high starch, low glucose potatoes through over-expression of the metabolic regulator, SnRK1. Plant Biotech J. 2006;4:409-18.

29. Kanegae H, Miyoshi K, Hirose T, Tsuchimoto S, Mori M, Nagato Y, Takano M. Expressions of rice sucrose non-fermenting-1 related protein kinase 1 genes 
are differently regulated during the caryopsis development. Plant Physiol Biochem. 2005;43:669-79.

30. Baena-González E, Rolland F, Thevelein JM, Sheen J. A central integrator of transcription networks in plant stress and energy signalling. Nature. 2007; 448:938-42.

31. Halford NG, Vicente-Carbajosa J, Sabelli PA, Shewry PR, Hannappel U, Kreis M. Molecular analyses of a barley multigene family homologous to the yeast protein kinase gene SNF1. Plant J. 1992;2:791-7.

32. Hannappel U, Vicente-Carbajosa J, Barker JHA, Shewry PR, Halford NG. Differential expression of two barley SNF1-related protein kinase genes. Plant Mol Biol. 1995;27:1235-40.

33. Takano M, Kajiya-Kanegae H, Funatsuki H, Kikuchi S. Rice has two distinct classes of protein kinase genes related to SNF1 of Saccharomyces cerevisiae, which are differently regulated in early seed development. Mol Gen Genet. 1998;260:388-94.

34. Halford NG. Regulation of carbon and amino acid metabolism: roles of sucrose nonfermenting-1-related protein kinase-1 and general control nonderepressible-2-related protein kinase. Adv Bot Res Inc Adv Plant Path. 2006:43:93-142.

35. Byrne EH, Prosser I, Muttucumaru N, Curtis TY, Wingler A, Powers S, Halford NG. Over-expression of GCN2-type protein kinase in wheat has profound effects on free amino acid concentration and gene expression. Plant Biotech J. 2012;10:328-40.

36. Foster R, Izawa T, Chua N-H. Plant bZIP proteins gather at ACGT elements. FASEB J. 1994;8:192-200.

37. Hummel M, Rahmani F, Smeekens S, Hanson J. Sucrose-mediated translational control. Ann Bot. 2009;104:1-7.

38. Müller M, Knudsen S. The nitrogen response of a barley C-hordein promoter is controlled by positive and negative regulation of the GCN4 and endosperm box. Plant J. 1993;4:343-55.

39. Schmidt RJ, Burr FA, Aukerman MJ, Burr B. Maize regulatory gene Opaque2 encodes a protein with a "leucine-zipper" motif that binds to zein DNA. Proc Natl Acad Sci U S A. 1990;87:46-50.

40. Pysh LD, Aukerman MJ, Schmidt RJ. OHP1: a maize basic domain/leucine zipper protein that interacts with Opaque2. Plant Cell. 1993;5:227-36.

41. Vicente-Carbajosa J, Moose SP, Parsons R, Schmidt RJ. A maize zinc-finger protein binds the prolamin box in zein gene promoters and interacts with the basic leucine zipper transcriptional activator Opaque2. Proc Natl Acad Sci U S A. 1997;94:7685-90.

42. Albani D, Hammond-Kosack MC, Smith C, Conlan S, Colot V, Holdsworth M, Bevan MW. The wheat transcriptional activator SPA: a seed-specific bZIP protein that recognizes the GCN4-like motif in the bifactorial endosperm box of prolamin genes. Plant Cell. 1997;9:171-84.

43. Halford NG, Hardie DG. SNF1-related protein kinases: global regulators of carbon metabolism in plants? Plant Mol Biol. 1998;37:735-48.

44. Chen Z, Muttucumaru N, Powers SJ, Halford NG. Expression analysis of abscisic acid (ABA) and metabolic signalling factors in developing endosperm and embryo of barley. J Cereal Sci. 2013;58:255-62.

45. Chang SJ, Puryea J, Cairney J. A simple and efficient method for isolating RNA from pine tree. Plant Mol Biol Rep. 1993;11:113-6.

46. Götz S, García-Gómez JM, Terol J, Williams TD, Nagaraj SH, Nueda MJ, Robles M, Talón M, Dopazo J, Conesa A. High-throughput functional annotation and data mining with the Blast $2 \mathrm{GO}$ suite. Nucleic Acids Res. 2008:36:3420-35.

\section{Publisher's Note}

Springer Nature remains neutral with regard to jurisdictional claims in published maps and institutional affiliations.

\section{Ready to submit your research? Choose BMC and benefit from:}

- fast, convenient online submission

- thorough peer review by experienced researchers in your field

- rapid publication on acceptance

- support for research data, including large and complex data types

- gold Open Access which fosters wider collaboration and increased citations

- maximum visibility for your research: over $100 \mathrm{M}$ website views per year

At BMC, research is always in progress.

Learn more biomedcentral.com/submissions 